\title{
NICE INFINITARY LOGICS
}

\author{
SAHARON SHELAH
}

\section{Contents}

0. Introduction for non-logicians

1. Introduction and preliminaries

2. The logic $\mathbb{L}_{\kappa}^{1}$

3. Serious properties of $\mathbb{L}_{\kappa}^{1}$

4. $\quad \mathbb{L}_{\kappa}^{1}$ is strong

\section{INTRODUCTION FOR NON-LOGICIANS}

The first part of the introduction tries to explain the aim to a general mathematical audience, so may be skipped by a knowledgeable reader; naturally we should start by explaining what is first order logic and a general (abstract) logic from a model-theoretic perspective.

We may consider classes of rings and classes of groups but usually we do not consider a class containing structures of both kinds. Formally a ring is a structure (or model) $M$ consistent with its universe, a set of elements called $|M|$ (but we may write $a \in M)$ and interpretations $+^{M}, \times^{M}$ and $0^{M}$ of the binary function symbols ,$+ \times$ and the individual constant symbol (= zero place function symbol) 0 . We also write ${ }^{n} M$ for $\left\{\left(a_{0}, \ldots, a_{n-1}\right): a_{0}, \ldots, a_{n-1}\right.$ are elements of $\left.M\right\}$.

Generally we have a so-called vocabulary $\tau$ consisting of relation symbols (= predicates) and function symbol $x$, each with a given number of places (= arity).

For a ring $M$ we consider many times the set of $n$-tuples satisfying some equations. Model theorists usually look at a wider class of such sets, which start with the family $\left\{\left\{\bar{a} \in{ }^{n} M: \bar{a}\right.\right.$ satisfies an equation $\left.\varphi\right\}: n \in \mathbb{N}$ and $\varphi$ an equation $\}$ and close it under intersection of two (with the same $n$ ), complements inside the relevant ${ }^{n}(M)$ and projection (from ${ }^{n+1} M$ to ${ }^{n} M$ ). So a first order formula for the vocabulary $\tau, \varphi=\varphi\left(x_{0}, \ldots, x_{n-1}\right)$ is a scheme giving for a $\tau$-structure $M$ a subset $\varphi(M)$ of ${ }^{n} M$ as above. If $n=0, \varphi(M) \in\{\{\langle\rangle\}, \emptyset\}$, then we call $\varphi$ a sentence and

Received by the editors May 16, 2010 and, in revised form, June 10, 2011.

2010 Mathematics Subject Classification. Primary 03C95; Secondary 03C80, 03C55.

Key words and phrases. Model theory, soft model theory, characterization theorems, Lindström theorem, interpolation, well ordering.

The author thanks Alice Leonhardt for the beautiful typing. The author thanks the Israel Science Foundation for partial support of this research. Part of this work was done while the author was visiting Mittag-Leffler Institut, Djursholm, Sweden, in the fall of 2000 and the fall of 2009. We thank the Institut for hospitality and support. Publication No. 797 in the author list of publications. 
say that $M$ satisfies it, $M \models \varphi$ iff $\varphi(M) \neq \emptyset$; let $\mathbb{L}(\tau)$ be the set of first order sentences or formulas in the vocabulary $\tau$; as we can add additional individual constants, the difference is minor. We may consider sets definable with parameters, i.e. $\varphi(M, \bar{b})=\{\bar{a}: M \models \varphi[\bar{a}, \bar{b}]\}$.

There is much to be said for first order logic; e.g. this family of subsets of ${ }^{n} M$ for $n \in \mathbb{N}$ has better closure properties than the set "solutions of finitely many equations", i.e. varieties; however, we shall not say it here. But in first order logic we cannot express, e.g. "a group $G$ is locally finite, i.e. every finitely generated subgroup is finite". To express this we may allow: if $\varphi_{k}\left(x_{0}, \ldots, x_{n-1}\right)$ is a formula for $k \in \mathbb{N}$, then so is $\varphi\left(x_{0}, \ldots, x_{n-1}\right)=\bigwedge_{k} \varphi_{k}\left(x_{0}, \ldots, x_{k}\right)$, i.e. $\varphi(M)=\bigcap\left\{\varphi_{k}(M)\right.$ : $k\}$.

Allowing this we get the logic $\mathbb{L}_{\aleph_{1}, \aleph_{0}}$; more generally,

$\boxplus_{0}$ the logic $\mathbb{L}_{\lambda, \kappa}$ is defined similarly but the formulas have the form $\varphi=$ $\varphi\left(\left\langle x_{i}: i<\gamma\right\rangle\right), \gamma<\kappa$ and we allow $\bigwedge_{\alpha<\beta} \varphi_{\alpha}(\bar{x})$ for $\beta<\lambda$ and

$$
\left(\exists y_{0}, \ldots, y_{\alpha}, \ldots\right)_{\alpha<l g(\bar{y})} \varphi(\bar{x}, \bar{y})
$$

where we allow a formula to have $<\kappa$ free variables; i.e. we consider subsets of ${ }^{\alpha} M$ for $\alpha<\kappa$ and allow intersection of $<\lambda$ (instead of two) and projections "forgetting" $<\kappa$ variables .

Another strengthening of first order logic is allowing $\psi(\bar{y})=\left(\exists \geq \aleph^{\aleph_{1}} x \varphi(x, \bar{y})\right)$, i.e. $\psi(\bar{M})=\{\bar{b}$ : for uncountably many $a \in M$ we have $M \models \varphi[b, \bar{a}]\}$. There are many other logics.

Now first order logic has many good properties, including (recall, the cardinality of a set $A$ is the number of elements, which may be infinite, and the cardinality of a model is the number of its elements, i.e. the cardinality of its universe $\|M\|)$

$\boxplus_{1}$ the downward LST (Löwenheim-Skolem-Tarski) property:

(a) if a sentence $\psi \in \mathbb{L}(\tau)$ has a model, i.e. $M \models \psi$, then it has a countable model;

(b) if $\tau$ is a vocabulary, $M$ is a $\tau$-model, $A \subseteq|M|,|A|+|\tau|+\aleph_{0} \leq \lambda<\|M\|$, then there is a $\tau$-model $N$ of cardinality $\lambda$, a submodel of $M$ such that $A \subseteq|N|$ and $\operatorname{Th}_{\mathbb{L}}(N)=\operatorname{Th}_{\mathbb{L}}(M)$, where

- $\operatorname{Th}_{\mathbb{L}}(M)=\left\{\psi \in \mathbb{L}\left(\tau_{M}\right): \psi\right.$ a sentence $\left.M \models \psi\right\}$.

This (together with the upward LST property) means that first order logic does not distinguish infinite cardinals.

$\boxplus_{2}$ compactness: if $T$ is a set of sentences in $\mathbb{L}(\tau)$ and every finite $T^{\prime} \subseteq T$ has a model, i.e. for some $\tau$-model $M$ we have $\varphi \in T^{\prime} \Rightarrow M=\varphi$, then $T$ has a model.

The desirability of this should be obvious.

$\boxplus_{3}$ interpolation: if $\tau_{0}=\tau_{1} \cap \tau_{2}$ are vocabularies, $\psi_{1} \in \mathbb{L}\left(\tau_{1}\right), \psi_{2} \in \mathbb{L}\left(\tau_{2}\right)$ and $\psi_{1} \vdash \psi_{2}$, i.e. there is no model of $\psi_{1} \wedge \neg \psi_{2}$, (equivalently if $M$ is a $\left(\tau_{1} \cup \tau_{2}\right)$ model and $M \models \psi_{1}$, then $\left.M \models \psi_{2}\right)$, then there is $\varphi \in \mathbb{L}\left(\tau_{0}\right)$ such that $\psi_{1} \vdash \varphi$ and $\varphi \vdash \psi_{2}$. 
First order logic satisfies interpolation: this is Craig's theorem. Lindström set out to show that first order logic is the natural choice, recalling there are many logics; for this he has first to define a logic, essentially (see more in Definition 1.9)

$\boxplus_{4}$ a logic $\mathscr{L}$ consists of the following:

(a) a set of sentences $\mathscr{L}(\tau)$ for any vocabulary $\tau$; we can define formulas $\varphi\left(x_{0}, \ldots, x_{n-1}\right)$ by adding to $\tau$ individual constants;

(b) satisfaction relation $\models \mathscr{L}$, i.e. $M \models \mathscr{L} \psi$, where $M$ a model, $\psi \in$ $\mathscr{L}\left(\tau_{M}\right)$

(c) natural properties such as preservation under isomorphisms and monotonicity (i.e. $\tau_{1} \subseteq \tau_{2} \Rightarrow \mathscr{L}\left(\tau_{1}\right) \subseteq \mathscr{L}\left(\tau_{2}\right)$ ).

This seems too wide, so (see more in Definition 1.12):

$\boxplus_{5} \mathscr{L}$ is a nice logic if the set of sentences $\mathscr{L}(\tau)$ has some natural closure properties such as:

- if $\psi_{1}, \psi_{2} \in \mathscr{L}(\tau)$, then for some $\psi \in \mathscr{L}(\tau)$ we have: $M \models \psi$ iff $M \models \psi_{1}$ and $M \models \psi_{2}$.

There is a natural order on the class of logics:

$\boxplus_{6} \mathscr{L}_{1} \leq \mathscr{L}_{2}$ iff for every vocabulary $\tau$ and $\psi_{1} \in \mathscr{L}_{1}(\tau)$ there is $\psi_{2} \in \mathscr{L}_{2}(\tau)$ such that: if $M$ is a $\tau$-model, then $M \models \mathscr{L}_{1} \psi_{1}$ iff $M \mid=\mathscr{L}_{2} \psi_{2}$;

$\boxplus_{7} \mathscr{L}_{1}, \mathscr{L}_{2}$ are equivalent if $\mathscr{L}_{1} \leq \mathscr{L}_{2}$ and $\mathscr{L}_{2} \leq \mathscr{L}_{1}$.

Now we can phrase (really just one of the versions 1 of

$\otimes$ (Lindström theorem) The logic $\mathscr{L}$ is equivalent to $\mathbb{L}$, first order logic when

(a) $\mathscr{L}$ is a nice logic;

(b) $\mathscr{L}$ satisfies LST to $\aleph_{0}$ i.e. $\boxplus_{1}(a)$;

(c) $\mathscr{L}$ satisfies compactness; see $\boxplus_{2}$.

This indicates that the family of nice logics not equivalent to $\mathbb{L}$ is the union of:

- the infinitary ones, usually above $\mathbb{L}_{\aleph_{1}, \aleph_{0}}$;

- the somewhat compact, usually $\aleph_{0}$-compact ones.

We here deal with the first.

Lindström's theorem founded "abstract model theory", where we have variables over logics. In the seventies and eighties this area flourished but a reason for its almost dying out is the lack of similar theorems for other logics, i.e. discovering (or pointing out) "interesting" logics which can be characterized in a reasonable way.

The aim of this work is to present such an infinitary logic and prove that it has some desirable properties. In particular it satisfies interpolation, which holds only in a "few" cases. This solves some more specific old problems, and we hope it will reopen the case of "abstract model theory".

In more detail, we define the logic $\mathbb{L}_{\kappa}^{1}$ for any suitable cardinal $\kappa$ playing the role of $\aleph_{0}$ in first order logic:

\footnotetext{
${ }^{1}$ E.g. compactness just for countable theorems, but then we have to add that the occurrence number is $\aleph_{0}$ or just $\aleph_{0}$; see Definition 1.11
} 
$\boxplus \mathbb{L}_{\kappa}^{1}$ satisfies

(a) a downward LST; any sentence which has a model has one of cardinality $<\kappa$;

(b) if the vocabulary has cardinality $<\kappa$, then the number of sentences is $\kappa$;

(c) a weak substitute of compactness: well ordering is not definable;

(d) the $\mathbb{L}_{\kappa}^{1}$-theory of a product of two $\tau$-models $M_{1} \times M_{2}$ depends just on the $\mathbb{L}_{\kappa}^{1}$-theories of $M_{1}$ and $M_{2}$;

(e) interpolation; see $\boxplus_{3}$.

\section{INTRODUCTION AND PRELIMINARIES}

1(A). Aims. We feel that this is an important one among my works and will attract little attention. Is this an oxymoron? We do not think so. See below.

The investigation of model-theoretic logics and soft model-theory started with Lindström theorems and was a central topic of model theory in the seventies. Major aims were to find characterization theorems, new important logics and non-trivial implications. The achievements were to a large extent summed up in the handbook of Barwise and Feferman [Be85], but then the subject became quite muted. There were some external reasons: stability theory and theoretical computer science draw people away, and there were also some incidental personal reasons. But probably the profound reason was a disappointment. The impression was that there were just too many examples and counterexamples but not enough deep results and, particularly, too many logics and too few characterization theorems (saying a logic $\mathbb{L}$ is the unique logic such that ...). Recall that Lindström characterized first order logic, e.g., as the only "reasonable" logic satisfying compactness (for $\aleph_{0}$ sentences) and the downward LST theorem (for one sentence, to $\aleph_{0}$ ), (but see $\S 1(\mathrm{D})$ below). Still there was some activity later, particularly of Väänänen.

Here we try to reopen the case. A property which remained mysterious was interpolation; see Makowsky Mak85] in the handbook. It was known that $\mathbb{L}_{\aleph_{1}, \aleph_{0}}$ has interpolation (Lopez-Escobar) but not $\mathbb{L}_{\lambda, \kappa}$ when $(\lambda, \kappa) \neq\left(\aleph_{0}, \aleph_{0}\right),\left(\aleph_{1}, \aleph_{0}\right)$ (Malitz). On $\mathbb{L}_{\kappa, \theta}$, see Dickman [Dic85]. However, the pair $\left(\mathbb{L}_{<\infty, \omega}, \mathbb{L}_{\infty, \infty}\right)$ and even $\left(\mathbb{L}_{\lambda^{+}, \omega}, \mathbb{L}_{\left(2^{\lambda}\right)^{+}, \lambda^{+}}\right)$has interpolation, a puzzling result. This leads naturally to a question: does this interpolation come from the existence of an intermediate logic which has interpolation? See more on the history of those questions and on interpolation and related subjects, Mak85.

Let us recall some old questions on which we do not advance here. Feferman raises the question

Question 1.1. Is there an $\aleph_{0}$-compact logic strenghtening $\mathbb{L}\left(\exists \geq \aleph_{1}\right)$ with interpolation.

Note the plethora of extensions of $\mathbb{L}\left(\exists \geq \aleph_{1}\right)$. For my taste preferable is

Question 1.2. 1) Is there a $\lambda$-compact logic stronger than first order satisfying interpolation, for any $\lambda$ ?

2) Moreover, a fully compact one?

Of course, part (2) became a question only after fully compact logics $>\mathbb{L}$ were discovered ([Sh:18] $)$. 
The introduction of $\mathrm{Be} 85$ mentions the (then latest advance): some compact logic strengthening first order logic satisfies the Beth definability theorem ([Sh:199]), a puzzling result. Also the pair $\left(\mathbb{L}\left(Q_{\aleph_{0}}^{\text {cf }}\right), \mathbb{L}(a a)\right)$ of logics satisfies interpolation, again a puzzling result. Those cases give hope of better results using related new logics. Returning to infinitary logics, the following old problems are (and will be) our main concern:

Problem 1.3. Is there a logic $\mathbb{L}$ satisfying interpolation such that $\mathbb{L}_{<\infty, \aleph_{0}} \subseteq \mathbb{L} \subseteq$ $\mathbb{L}_{<\infty,<\infty}$ ?

Problem 1.4. Assume $\kappa$ is strong limit singular of cofinality $\aleph_{0}$. Is there a logic $\mathbb{L}$ satisfying interpolation such that $\mathbb{L}_{\kappa^{+}, \aleph_{0}} \subseteq \mathbb{L} \subseteq \mathbb{L}_{\kappa^{+}, \kappa}$ ?

Later we have asked ourselves:

Problem 1.5. Is there, for an arbitrarily large cardinal $\kappa$, a logic $\mathbb{L}$ such that:

(a) $\mathbb{L}_{\kappa, \omega} \subseteq \mathbb{L}$ and has reasonable closure properties;

(b) $\mathbb{L}$ has the downward LST property in the sense that every sentence which has a model $N$, has a model $N$ of "small" cardinality; moreover, $M \subseteq N$;

(c) $\mathbb{L}$ has interpolation;

(d) undefinability of well ordering (in a strong sense) which means: if $M$ expands $(\mathscr{H}(\lambda), \in)$ and $M \models \psi$, then for some $N$ we have

( $\alpha) \quad N \models \psi$,

$(\beta) \operatorname{ord}^{N}$ is not well founded.

A posteriori we add

$(\gamma)|N|$ is the union of $\aleph_{0}$, an internal set of bounded cardinality; i.e. for some $\left\langle a_{n}: n<\omega\right\rangle, \theta, N \models$ " $\theta$ a cardinal such that $\left|a_{n}\right| \leq \theta$ and $(\forall b \in N)\left(\bigvee_{n<\omega} N \models " b \in a_{n}\right) " ;$

(e) $\mathbb{L} \subseteq \mathbb{L}_{\theta, \theta}$ for a suitable $\theta$.

Problem 1.6. Is there a maximal such logic?

There is a feeling that Lindström's theorem, EF-games and interpolation are inherently connected; though I do not know of a formalization of it, the present work gives evidence strengthening this feeling.

1(B). What is achieved. We feel that here we reasonably fulfill those old hopes mentioned above, in the direction of non-compact logics (recall that by the Lindström theorem, any (nice) logic stronger than first order logic fails downward LST to $\aleph_{0}$ or fails $\aleph_{0}$-compactness).

Assuming for transparency $\kappa=\beth_{\kappa}$, we find an interesting logic, $\mathbb{L}_{\kappa}^{1}$, such that:

$\circledast_{1}(A) \quad \mathbb{L}_{\kappa}^{1}$ is a nice logic.

$(B)$ It has a reasonable characterization: it is the maximal nice logic, see Definitions 1.9, 1.12, such that $(\alpha,<)$ can be characterized up to isomorphism by some $\psi_{\alpha} \in \mathbb{L}_{\kappa}^{1}$ for $\alpha<\kappa$, and it has occurrence number $\leq \kappa$, see Definition 1.11, and well ordering is not definable in a strong way.

(C) It satisfies interpolation 2 (see $\boxplus_{3}$ above or Definition 1.14 below, answering an old question on the existence of such a logic).

\footnotetext{
${ }^{2}$ For consequences of interpolation, see Mak85.
} 
(D) It is between $\mathbb{L}_{<\kappa}^{-1}=\bigcup\left\{\mathbb{L}_{\lambda^{+}, \aleph_{0}}: \lambda<\kappa\right\}$ and

$\mathbb{L}_{<\kappa}^{0}=\bigcup\left\{\mathbb{L}_{\lambda^{+}, \lambda^{+}}: \lambda<\kappa\right\}$; see $\boxplus_{0}$ above or Definition 1.20 below.

(E) It has many of the good properties of $\mathbb{L}_{\kappa}^{0}:=\mathbb{L}_{<\kappa}^{0}$ :

$(\alpha)$ downward LST, see $\boxplus_{1}$ above, specifically every $\psi \in \mathbb{L}_{\kappa}^{1}(\tau)$ has a model of cardinality $<\kappa$ (see some variants in Definition 2.14 below);

( $\beta) \quad$ well ordering is not an expressibl 3 ;

$(\gamma)$ addition of theories; see Theorem 3.14 .

$(\delta)$ product of (two) theories; see Theorem 3.14

$(F) \quad$ Alternative characterization: $\mathbb{L}_{\kappa}^{1}$ is a minimal nice logic $\mathscr{L}$ for which

- for any ordinal $\alpha<\kappa$ we can characterize $(\alpha,<)$ up to isomorphism by some $\psi_{\alpha} \in \mathscr{L}$;

- $\quad$ we can characterize the class of $(A \cup \mathscr{P}, A, \in)$, where $\mathscr{P} \subseteq[A] \leq \mu$ is an $\aleph_{0}$-cover (and $A \cap \mathscr{P}=\emptyset$ ) for each $\mu<\kappa$ by some sentence from $\mathscr{L}$ (see Definition 3.6);

- $\mathscr{L}$ is $\Delta$-closed; see Definition 3.8 .

- $\mathscr{L}$ has occurrence number $\leq \kappa$; see Definition 1.11.

We do not have a generalization of the Feferman-Vaught theorem [FV59] on general operations and not even the Mostowski one, Mos52, on reduced products, even for the product of countably many models; see Theorem 4.10.

Here in $\S 2$ we choose a definition of the logic closest to the way we arrive at it and at the proof. For $\alpha, \theta<\kappa$ we generalize the Ehrenfuecht-Fraisse game allowing "rescheduling of debts". This does not give an equivalence relation, so we close the induced relation to an equivalence relation and a sentence; i.e. the class of models of a sentence is the union of some such equivalence classes. Then we prove the basic properties.

In $\S 3$ we deal with the deeper properties as promised in $\circledast_{1}$ above: non-definability of well ordering, characterization and interpolation.

In $\S 4$ we show how close is our logic to $\mathbb{L}_{\kappa}^{0}$ and deal with sums and products. We intend to continue in [Sh:F1046], in particular concerning Problem 1.4.

More than once, lecturing on this, some in the audience "complain" that this definition does not sound like a definition of logic. So in [Sh:F1046] we intend to give a presentation close to the way logics are traditionally defined (we could have done it for $\mathbb{L}_{\kappa}^{1}$, too) but our characterization theorem shows that we shall get the same logic.

The logic $\mathbb{L}_{\kappa}^{1}$ from $\S 1$ is quite satisfactory: many of the good properties of $\mathbb{L}_{<\kappa, \aleph_{0}}$ and interpolation, and a characterization (parallel to the Lindström theorem). But compared to $\mathbb{L}_{<\kappa, \aleph_{0}}$ we lose the upward LST.

Question 1.7. Letting $\kappa=\beth_{\kappa}$, is there an $\mathbb{L}$ such that $\mathbb{L}_{\kappa}^{-1} \leq \mathscr{L} \leq \mathbb{L}_{\kappa}^{0}$ satisfying interpolation and the upward LST theorem?

We intend to deal with this in [Sh:F1046].

\footnotetext{
${ }^{3}$ That is, if $\psi$ is a sentence and for every ordinal $\alpha$ for some $M, M \models \psi$ and $\left(P^{M},<^{M}\right)$ is a well ordering of order type $\geq \alpha$, then for some model $M$ of $\psi,\left(P^{M},<^{M}\right)$ is not a well ordering.
} 
1(C). Why characterizations? Note that characterization theorems are central for several reasons:

$\circledast_{2}(a) \quad$ per se, uniqueness results are nice, of course;

(b) historically, the Lindström theorem has this form;

(c) they prove that a logic is a natural logic;

(d) for a logic which lacks such a theorem we may well suspect that

- there are many relatives of similar good properties, without a special reason to prefer one or another.

How good is a characterization theorem? Of course, it all depends on the properties appearing in the characterization being natural and preferably well established.

The situation of having reasonable logics which we can strengthen while preserving their main positive properties but neither seeing a maximal one nor proving such an extension does not exist has been prominent in the area, e.g., the most well-established ones such as $\mathbb{L}(Q)=\mathbb{L}\left(\exists \geq \aleph^{\aleph_{1}}\right)$ and also $\mathbb{L}_{\kappa^{+}, \aleph_{0}}$.

Why here do we tend to look at strong limit cardinals, in particular, $\kappa=\beth_{\kappa}$ ? Note that in a first order formula with a fixed finite vocabulary, with predicates only (for transparency), the number of sentences of quantifier depth $q$ has order of magnitude $\beth_{q}$, iterated power of $q$ times. In infinitary logic, if we like that still there is a sentence expressing the "quantifier depth $\leq \alpha$ theory", we need $\kappa=\beth_{\kappa}$. A price is that for $\kappa$ singular, we lose full substitution, and moreover, full closure under conjunctions of $<\kappa$. This is resolved if we demand that $\kappa$ is a strong limit regular, i.e. (strongly) inaccessible, that is fine, but the existence of such cardinals is unprovable in $\mathrm{ZFC}$.

1(D). Directions ignored here. We do not deal here with some other major directions:

$\circledast_{3}(a) \quad \aleph_{0}$-compact (nice logic);

(b) logics without negation and continuous logic;

(c) almost isomorphism (and absolute logic).

We may look at model theory, essentially replacing "isomorphic" by "almost isomorphic", that is, isomorphisms by potential isomorphisms, i.e. isomorphism in some forcing extension. In Sh:12] we have suggested reconsidering a major theme in model theory, counting the number of isomorphism types. We call $M, N$ almostisomorphic when $M, N$ have (the same vocabulary and) the same $\mathbb{L}_{\infty, \aleph_{0}}$-theory, equivalently isomorphic in some generic extension. For a theory $T$ let $\mathbb{I}_{\mathrm{ai}}(\lambda, T)$ be $\mid\left\{M / \equiv_{\mathbb{L}_{\infty, \aleph_{0}}}: M\right.$ a model of $T$ of cardinality $\left.\lambda\right\} \mid$. This behaves nicely: if $T$ has cardinality $\leq \lambda$, is first order or just $\subseteq \mathbb{L}_{\lambda^{+}, \aleph_{0}}$, then $\dot{\mathbb{I}}_{\mathrm{ai}}(\lambda, T) \leq \lambda<\mu \Rightarrow \dot{\mathbb{I}}_{\mathrm{ai}}(\mu, T) \leq$ $\dot{\mathbb{I}}_{\mathrm{ai}}(\lambda, T)$ (on $\dot{\mathbb{I}}_{\mathrm{ai}}(-, T)$ for $\aleph_{0}$-stable $T$ : see a work of Laskowski-Shelah in preparation). In [Sh:12] we also define " $M$ is ai-rigid, i.e. $a \neq b \in M \Rightarrow(M, a) \not \equiv_{\mathbb{L}_{\infty, \aleph_{0}}}$ $(N, a) "$ and have the downward LST theorem for it. Later Nadel suggested further to consider homomorphisms, in particular for abelian groups; see EM02, Ch. IV, $\S 3$, p. 487], more Göbel-Shelah GbSh:880, Göbel-Herden-Shelah GbHeSh:948]. Barwise characterized the relevant logic, $\mathbb{L}_{\infty, \aleph_{0}}$, by absoluteness: (among logics with satisfaction being absolute under forcing it is maximal). 
1(E). Can soft model theory be applied? What about applications of soft model theory? There are some applications using compact logics. Concerning 1(D), 1(E) on other directions see [Be85]. See [Sh:384] on extending first order logic by second order quantifiers restricted in some ways. Of course, the expressive power of the logic depends on the restriction; see the examples below. In cofinality logic, $\mathbb{L}\left(\mathbf{Q}_{C}^{\text {cf }}\right)$ with $\mathbf{C}$ a class of regular cardinals, we are allowed to say: the formula $\varphi(x, y)$, possibly with parameters, defines a linear order with no last element of cofinality from $\mathbf{C}$, recalling that the cofinality of a linear oder $I$ is the minimal cardinality of an unbounded (equivalently cofinal) subset. If $\mathbf{C}$ is non-trivial, this logic is a very interesting logic (e.g., fully compact), in particular showing what we cannot prove, so full compactness is not sufficient to characterize first order logic. But its expressive power is weak, so we do not expect it to have applications.

In Sh:384 (where you can find something about the history of the topic) we prove the compactness of the quantifier (that is, for first order logic extended by it) $\mathscr{L}^{\text {ceab }}=\mathbb{L}\left(\mathbb{Q}^{\text {ceab }}\right)$, quantifying over complete embeddings of one atomless Boolean ring into another. Moreover, for this logic we prove completeness for a natural set of axioms. Now consider the problem "can the automorphism groups of a 1homogeneous 4 Boolean algebra be non-simple?" 5 Much is known about this group and, in particular, that it is "almost" simple; see Rubin-Štěpánek [Rv89]. It was known that there may exist such Boolean Algebras, as by [Sh:b, IV] in some generic extension, all automorphisms of $\mathscr{P}(\omega)$ /finite are trivial (i.e. induced by permutations $\pi$ of $\mathbb{Z}$ such that $\{n \in \mathbb{Z}: n \geq 0$ but $\pi(n)<0\}$ is finite) and van Dowen notes that the group of trivial automorphisms of $\mathscr{P}(\omega)$ /finite is not simple (as the subgroup of the automorphisms induced by permutations of $\omega$ is a normal subgroup) and the quotient is isomorphic to $(\mathbb{Z},+)$. Alternatively, Koppelberg Kop85 has directly constructed such Boolean Algebras of cardinality $\aleph_{1}$ assuming (the more natural assumption) $\mathrm{CH}$. So by the completeness theorem (as the set of axioms is absolute), as the relevant facts are expressible in $\mathbb{L}\left(\mathbb{Q}^{\text {ceab }}\right)$, the existence is proved in ZFC. Some may want to consider a direct proof. It almost certainly will give more specific desirable information.

Another helpful quantifier is on branches of trees (see [Sh:72]). In Fuchs-Shelah FuSh:766] it is used to eliminate the use of diamonds, i.e. to prove in ZFC the existence of valuation domains $R$ such that there are $R$-modules which are universal (i.e., the family of submodules is linearly ordered by inclusion) but not standard. Note the obvious examples (which are called standard): $R$ itself or appropriate quotients. Actually the completeness theorem for this logic gives an absoluteness result which is used.

We believe that quantifiers with completeness and compactness will be useful, so it is worthwhile to find such quantifiers. Hopefully, we will see more in [Sh:800].

\section{1(F). Preliminaries.}

Notation 1.8. 1) $\tau$ denotes a vocabulary, i.e. a set of predicates and function symbols so that each $P \in \tau$ has $\operatorname{arity}_{\tau}(P)<\omega$ places and each function symbol $F \in \tau$ has $\operatorname{arity}_{\tau}(F)<\omega$ places; of course, individual constants are zero-place

\footnotetext{
${ }^{4} \mathrm{~A}$ Boolean algebra $B$ is 1 -homogeneous if it is atomless and for every $a, b \in B \backslash\left\{0_{\mathbb{B}}\right\}$, we have $B \cong B \nmid b$ (equivalently for $a, b \in \mathbb{B} \backslash\left\{0_{B}, 1_{B}\right\}$ for some automorphism $f$ of $\left.B, f(a)=b\right)$.

${ }^{5}$ That is, it has no normal subgroup which is neither the full group nor the one-element subgroup.
} 
function symbols and we may write $\operatorname{arity}(P), \operatorname{arity}(F)$ when $\tau$ is clear from the context.

1A) For a structure $M$ let $\tau_{M}$ be the vocabulary of $M$; for a predicate $P$ from $\tau_{M}, P^{M}$ is the interpretation of $P$, so an $\operatorname{arity}_{\tau}(P)$-place relation on $|M|$, the universe of $M$; similarly for a function symbol $F$ from $\tau$ and in particular for an individual constant $c$ from $\tau$.

2) $\mathscr{L}$ denotes a logic; see Definition 1.9 .

3) $\bar{x}, \bar{y}, \bar{z}$ denote sequences of variables (with no repetition). Usually $\bar{x}=\left\langle x_{i}\right.$ : $i\langle\alpha\rangle$. so $\alpha=\ell g(\bar{\alpha})$ but even possibly $\bar{x}=\left\langle x_{s}: s \in S\right\rangle$ and then we let $\ell g(\bar{x})=S$.

4) We say that $\tau$ is a relational vocabulary when it has no function symbol.

Recall

Definition 1.9. 1) A logic $\mathscr{L}$ consists of

(a) function $\tau \mapsto \mathscr{L}(\tau)$ giving a set of sentences $\varphi$ (or formulas $\varphi(\bar{x})$; see Convention 1.10 below) for any vocabulary $\tau$; the function is a class function that is a definition;

(b) $\models \mathscr{L}$, satisfaction, i.e. the relation $M \models \mathscr{L} \varphi$ for $M$ a model, $\varphi \in \mathscr{L}\left(\tau_{M}\right)$;

(c) renaming: the function $\hat{\pi}$, depending on $\left(\tau_{1}, \tau_{2}, \pi, \mathscr{L}\right)$, is a one-to-one function from $\mathscr{L}\left(\tau_{1}\right)$ onto $\mathscr{L}\left(\tau_{2}\right)$ when $\pi$ is an isomorphism from the vocabulary $\tau_{1}$ onto the vocabulary $\tau_{2}$ (i.e. if $P \in \tau_{1} \Rightarrow$, then $\pi(P) \in \tau_{2}$ is a predicate and $\operatorname{arity}_{\tau_{1}}(P)=\operatorname{arity}_{\tau_{2}}(\pi(P))$ and similarly for $\left.F \in \tau_{1}\right)$;

(d) if $\pi$ is an isomorphism from the vocabulary $\tau_{1}$ onto the vocabulary $\tau_{2}$ and $M_{1}$ is a $\tau_{1}$-model and $M_{2}=\pi\left(M_{1}\right)$ is naturally defined, then $\varphi \in$ $\mathscr{L}\left(\tau_{1}\left(M_{1}\right)\right) \Rightarrow\left[M_{1} \models \varphi \Leftrightarrow M_{2} \models \hat{\pi}(\varphi)\right] ;$

(e) (isomorphism): if $M_{1}, M_{2}$ are isomorphic $\tau$-models and $\varphi \in \mathscr{L}(\tau)$, then $M_{1} \models \mathscr{L} \varphi \Leftrightarrow M_{2} \models \mathscr{L} \varphi$;

(f) (monotonicity): if $\tau_{1} \subseteq \tau_{2}$, then $\mathscr{L}\left(\tau_{1}\right) \subseteq \mathscr{L}\left(\tau_{2}\right)$ and for any $\tau_{2}$-model $M_{2}$ and $\varphi \in \mathscr{L}\left(\tau_{1}\right)$ we have $M_{2} \models \varphi \Leftrightarrow\left(M_{2} \mid \tau_{1}\right) \models \varphi$.

Convention 1.10. We define a formula $\varphi=\varphi(\bar{x})$ in $\mathscr{L}(\tau)$ as a sentence in $\mathscr{L}(\tau \cup$ $\left.\left\{c_{i}: i<\ell g(\bar{x})\right\}\right)$ with $c_{i}(i<\alpha)$ pairwise distinct individual constants not from $\tau$ and if $\varphi(\bar{x}) \in \mathscr{L}(\tau)$ and $\bar{a} \in{ }^{\ell g(\bar{x})} M$, then $M \models \mathscr{L} \varphi[\bar{a}]$ means $M^{+} \models \mathscr{L} \varphi$, where $M^{+}$is the expansion of $M$ by $c_{i}^{M^{+}}=a_{i}$ for $i<\ell g(\bar{x})$; in fact, $\ell g(\bar{x})$ can be any index set.

Definition 1.11. For a logic $\mathscr{L}$, the occurrence number oc $(\mathscr{L})$ of $\mathscr{L}$ is the minimal cardinal $\kappa($ or $\infty)$ such that $\mathscr{L}(\tau)=\bigcup\left\{\mathscr{L}\left(\tau^{\prime}\right): \tau^{\prime} \subseteq \tau\right.$ is of cardinality $\left.<\kappa\right\}$ for any vocabulary $\tau$.

Definition 1.12. We say that a logic $\mathscr{L}$ is nice when:

(a) ( $\alpha$ ) Applying predicates: if $P \in \tau$ is an $n$-place predicate, then $P\left(x_{0}, \ldots, x_{n-1}\right) \in \mathscr{L}(\tau)$ is a formula of $\mathscr{L}(\tau)$.

( $\beta) \quad$ Equality: $x_{0}=x_{1} \in \mathscr{L}(\tau)$; i.e. for any individual constants $c_{0}, c_{1} \in \tau$ there is $\varphi \in \mathscr{L}(\tau)$ such that $M \models \varphi$ iff $c_{0}^{M}=c_{1}^{M}$.

$(\gamma)$ Applying6 functions: for an $n$-place function symbol $F \in \tau$, $x_{0}=F\left(x_{1}, \ldots, x_{n-1}\right)$ is a formula of $\mathscr{L}(\tau)$.

\footnotetext{
${ }^{6}$ We may put together clauses $(a)(\alpha)$ and $(a)(\gamma)$ allowing $P\left(\sigma_{0}(\bar{x}), \ldots, \sigma_{n-1}(\bar{x})\right), \sigma_{\ell}$ a term; the choice is immaterial.
} 
(b) $(\alpha) \quad \mathscr{L}$ is closed under conjunction, i.e. for every $\varphi_{1}, \varphi_{2} \in \mathscr{L}(\tau)$ there is $\varphi_{3} \in \mathscr{L}(\tau)$ such that for every $\tau$-model $M$ we have:

$M \models \varphi_{3}$ iff $M \models \varphi_{1}$ and $M \models \varphi_{2}$.

(B) $\mathscr{L}$ is closed under existential quantifier, $(\exists x)$, i.e. for any $\varphi \in \mathscr{L}(\tau \cup$ $\{c\}), c$ an individual constant not in $\tau$, there is $\psi \in \mathscr{L}(\tau)$ such that for any $\tau$-model $M$ we have $M \models \varphi$ iff $M^{+} \models \varphi$ for some $(\tau \cup\{c\})$-expansion $M^{+}$of $\mu$.

$(\gamma) \quad \mathscr{L}$ is closed under negation, i.e. for any $\varphi \in \mathscr{L}(\tau)$ there is $\psi \in \mathscr{L}(\tau)$ such that for any $\tau$-model $M, M \not \models \mathscr{L} \varphi \Leftrightarrow M \models \mathscr{L} \psi$.

(c) Restricting a sentence $\psi$ to a predicate $P, \psi \uparrow P \in \mathscr{L}(\tau)$ when $\psi \in \mathscr{L}(\tau)$, where $P \in \tau$ is a unary predicate, and $\tau$ is a relational vocabulary; see Definition 1.15, Notation 1.8(4).

(d) Weak substitution, that is, has substitution for weak schemes; see Definition 1.17 below.

Remark 1.13. 1) Above we prefer $(a)(\gamma)$ on using $R^{\prime}\left(\sigma_{0}, \ldots, \sigma_{m-1}\right)$, where each $\sigma_{\ell}=\sigma_{\ell}(\bar{x})$ is a term.

2) Below we can define the multi-sort version.

Definition 1.14. 1) A logic $\mathscr{L}$ satisfies interpolation when for any sentence $\psi_{1} \in$ $\mathscr{L}\left(\tau_{1}\right), \varphi_{2} \in \mathscr{L}\left(\tau_{2}\right)$ and $\tau=\tau_{1} \cap \tau$, we have: if $\varphi_{1} \vdash \varphi_{2}$, i.e. for any $\left(\tau_{1} \cup \tau_{2}\right)$-model $M, M \models \varphi_{1} \Rightarrow M \neq \varphi_{2}$, then for some sentence $\psi \in \mathscr{L}(\tau)$ we have $\varphi_{1} \vdash \psi$ and $\psi \vdash \varphi_{2}$.

2) The natural definition for multi-sort languages.

Definition 1.15. 1) We say that $\varphi \equiv \psi\lceil P$, where $\varphi, \psi \in \mathscr{L}(\tau)$ or pedantically 7 $\varphi=\psi \uparrow_{\tau} P$, where $P$ is a unary predicate in the vocabulary $\tau$ and $\tau$ is of minimal cardinality such that $\psi \in \mathscr{L}(\tau) \underline{\text { when }}$ :

for any $\tau$-models $M, M \models\left(\psi \uparrow_{\tau} P\right)$ iff $P^{M}$ is non-empty, closed under $F^{M}$ for every function symbol from $\tau$ and $M \uparrow \overline{P^{M}} \models \psi$; see below.

2) For a $\tau$-model $M$ and unary predicate $P$ let $N=M\left\lceil P^{M}\right.$ be the $\tau$-model with universe $P^{M}$, for $n$-place predicate $Q \in \tau$ we have $Q^{N}=Q^{M} \cap^{n}|N|$ and for $n$-place function symbol $F \in \tau, F^{N}(\bar{a})=b \Leftrightarrow \bar{a} \in{ }^{n}|N| \wedge b \in N \wedge F^{M}(\bar{a})=b$.

Definition 1.16. Let $\mathscr{L}$ be a logic.

$0)$ at $\mathscr{L}(\tau)$ is the set of atomic formulas $\varphi(\bar{x})$ from Definition $1.12($ a); bs $\mathscr{L}(\tau)=$ $\{\varphi, \neg \varphi: \varphi \in$ at $\mathscr{L}(\tau)\}$; we may omit $\mathscr{L}$ if it is clear from the context.

1) Let $\varphi \vdash \mathscr{L} \psi$, where $\varphi, \psi \in \mathscr{L}(\tau)$ mean that $M \models \varphi \Rightarrow M \models \psi$ for any $\tau$-model (this does not depend on $\tau$ by Definition 1.9).

2) We say that $\bar{\vartheta}$ is an $\left(\mathscr{L}, \tau_{1}, \tau_{2}\right)$-interpretation scheme when:

(a) $\mathscr{L}$ is a logic.

(b) $\tau_{1}, \tau_{2}$ are vocabularies.

(c) $\bar{\vartheta}=\left\langle\vartheta_{\varphi(\bar{x})}(\bar{x}): \varphi(\bar{x}) \in \operatorname{at} \mathscr{L}\left(\tau_{2}\right)\right\rangle$.

(d) $\vartheta_{\varphi(\bar{x})}(\bar{x})$ is a formula in $\mathscr{L}\left(\tau_{1}\right)$, so $\varphi=P\left(x_{0}, \ldots, x_{n-1}\right), \bar{x}=\left\langle x_{\ell}: \ell<n\right\rangle$ or $\varphi=\left(x_{0}=F\left(x_{1}, \ldots, x_{n}\right)\right), \bar{x}=\left\langle x_{\ell}: \ell \leq n\right\rangle$.

${ }^{7}$ Of course $\psi\lceil\tau$ is not uniquely determined. We may like to be more liberal in restricting a model; in Notation 1.8 (1) allow $F^{M}$ to be a partial function for $F$, a function symbol from $\tau(M)$. We may combine this with restriction (see Definition 1.15). Then we still have to demand $P^{M} \neq \emptyset$ (except if we go further and allow empty models). Note that $M \models$ “ $\neg F(\bar{a})=b$ " when $F^{M}(\bar{a})$ is not well defined. 
2A) Above we say that the $\left(\left(\mathscr{L}_{1}, \tau_{1}, \tau_{2}\right)\right.$-interpretation $)$ scheme $\bar{\vartheta}$ is simple when $\vartheta_{x_{0}=x_{1}}$ has the form $\left(x_{0}=x_{1}\right)$.

3) For $\bar{\vartheta}$ as above we say that $N=N_{\bar{\vartheta}}[M]=M_{1}[\bar{\vartheta}]$ when

(a) $|N|=|M| / \varphi_{=}\left(x_{0}, x_{1}\right)$, which means that

- $E_{\varphi=}^{M}=\{(a, b): M=\varphi(a, b)\}$ is an equivalence relation on $|M|$ or just some non-empty subset;

- $|N|=\left\{a / E_{\varphi=}^{M}: a \in M\right.$ and $\left.M \models \varphi=[a, a]\right\}$.

(b) $N \models \varphi[\bar{a}] \Leftrightarrow M \models \vartheta_{\varphi(\bar{x})}[\bar{a}]$ for $\varphi(\bar{x}) \in$ at $\left(\tau_{2}\right)$ and $\bar{a} \in{ }^{\omega>} M$ of length $\ell g(\bar{x})$.

(Note: not for every such $\bar{\vartheta}$ and $\tau_{1}$-model $M_{1}$ is $N_{\bar{\vartheta}}\left[M_{2}\right]$ well defined. We need that $\varphi=(-,-)$ defines an equivalence relation on $\left|M_{1}\right|$, which is a congruence relation for the $\tau_{2}$-relations and functions we define and, of course, the definition of the functions gives a function, similarly below; but if $\bar{\vartheta}$ is simple this problem does not arise.)

4) We say that the logic $\mathscr{L}$ satisfies full substitution 8 when: if $\tau_{1}, \tau_{2}$ are vocabularies, $\bar{\vartheta}=\left\langle\vartheta_{\varphi(\bar{x})}(\bar{x}): \varphi(\bar{x}) \in\right.$ at $\left.\mathscr{L}\left(\tau_{2}\right)\right\rangle$ is a simple $\left(\bar{L}, \tau_{1}, \tau_{2}\right)$-interpretation scheme, and $\psi_{2} \in \mathscr{L}\left(\tau_{2}\right)$, then there is $\psi_{1} \in \mathscr{L}\left(\tau_{1}\right)$ such that: if $M_{1}=N_{\bar{\vartheta}}\left[M_{2}\right]$, see below, so $M_{\ell}$ is a $\tau_{\ell}$-model for $\ell=1,2$, then $M_{1} \models \psi_{1} \Leftrightarrow M_{2} \models \psi_{2}$.

$5)$ We say that the logic $\mathscr{L}$ satisfies substitution when we require $\bar{\vartheta}$ to be simple.

Definition 1.17. 1) We say that $\bar{\vartheta}$ is a weak $\left(\mathscr{L}, \tau_{1}, \tau_{2}\right)$-scheme (but $\mathscr{L}$ is immaterial so can be omitted) when:

(a) $\tau_{1}, \tau_{2}$ are vocabularies.

(b) $\bar{\vartheta}=\left\langle\vartheta_{\varphi(\bar{x})}(\bar{x}): \varphi(\bar{x}) \in \operatorname{at}\left(\tau_{2}\right)\right\rangle$.

(c) The formulas $\vartheta_{\varphi(\bar{x})}$ are atomic (or the conjunction of two atomic for equality) formulas in which we substitute some variables by individual constants; moreover:

$(\alpha)$ If $\varphi(\bar{x})$ is equal to $\left(x_{0}=x_{1}\right)$, so $\ell g(\bar{x})=2$, then $\vartheta_{\varphi(\bar{x})}=\left(x_{0}=\right.$ $x_{1} \wedge P_{\varphi(\bar{x})}\left(x_{0}, \bar{c}_{\varphi(\bar{x})}\right)$.

( $\beta)$ If $\varphi(\bar{x})=P(\bar{x})$, so $P \in \tau_{2}$ is a predicate, then

$\bullet_{1} \quad \vartheta(\bar{x})=Q_{\varphi(\bar{x})}(\bar{x}, \bar{c})$ where,

$\bullet_{2} Q_{\varphi(\bar{x})}$ is a predicate from $\tau_{1}$,

- $3 \quad \bar{c}$ is a sequence of individual constants from $\tau_{1}$,

- $4 \quad$ so $\operatorname{arity}_{\tau_{2}}(P)=\ell g(\bar{x}), \operatorname{arity}_{\tau_{1}}\left(Q_{p}\right)=\ell g(\bar{x})+\ell g(\bar{c})$.

$(\gamma)$ If $\varphi(\bar{x})=\left(x_{0}=F\left(x_{1}, \ldots, x_{n}\right)\right)$, so $F \in \tau_{2}$ is a function symbol, then

$\bullet_{1} \vartheta_{\varphi(\bar{x})}(\bar{x})$ is $\left(x_{0}=H_{F}\left(x_{1}, \ldots, x_{n}, \bar{c}_{F}\right)\right)$ or $Q_{F}\left(x_{0}, \ldots, x_{n}, \bar{c}_{F}\right)$,

$\bullet_{2}, \bullet_{3}$ as above.

2) We say that $\mathscr{L}$ has weak substitution when $\mathscr{L}$ satisfies Definition 1.16 (4), but restricting ourselves to weak schemes.

Remark 1.18. 1) The meaning of Definition $1.17(1)(\mathrm{c})(\gamma)$ is

$(*)_{1} \bullet \vartheta_{\varphi(\bar{x})}(\bar{x})=\left(x_{0}=H_{F}\left(x_{1}, \ldots, x_{n}, \bar{c}_{F}\right)\right) ;$

- $H_{F} \in \tau_{1}$ is a function symbol of arity $n+\ell g\left(\bar{c}_{F}\right)$;

- $\bar{c}_{F}$ is a sequence of individual constants

${ }^{8}$ We do not use full substitution, as for $\kappa$ singular, $\mathbb{L}_{\kappa}^{0}, \mathbb{L}_{\kappa}^{1}$ are not closed under full substitution. 
or

$(*)_{2}$ similarly using $Q_{F}\left(x_{0}, \ldots, x_{n}, \bar{c}_{F}\right)$.

2) Why in Definition 1.17 (1)(c) do we need the "moreover"; i.e. why is the second version stronger? As we demand the $\bar{c}_{\varphi}$ 's to be as in the end, it does not matter whether we ask it or not. We could further demand that $\bar{c}_{\varphi}=\left\langle c_{*}\right\rangle$ for all $\bar{\varphi} \in \operatorname{at}\left(\tau_{2}\right)$.

$3)$ In the "weak $\left(\mathscr{L}, \tau_{1}, \tau_{2}\right)$-scheme" (hence in "weak substitution)" we may use first order formulas (instead of atomic); that is, our results will not be affected by this change in the definition.

4) Also, in the "weak $\left(\mathscr{L}, \tau_{1}, \tau_{2}\right)$-scheme" we may add $\bar{c}_{\varphi}=\left\langle c_{*}\right\rangle$, for one $c_{*}$, and/or demand that $\bar{\vartheta}$ is simple. For the latter change in the proof of Theorem 3.4 . we have to say that "without loss of generality, all $\left\|M_{\alpha, n}\right\|$ have cardinality $\geq \mu_{1}$ and in fact $=\mu_{1}$ "; for this we need a suitable version of LST, so have to add the assumption " $\mathscr{L}$ satisfies the suitable version of LST".

Definition 1.19. 1) For logics $\mathscr{L}_{1}, \mathscr{L}_{2}$ let $\mathscr{L}_{1} \subseteq \mathscr{L}_{2}$ mean that: $\mathscr{L}_{1}(\tau) \subseteq \mathscr{L}_{2}(\tau)$ for any vocabulary $\tau$ and $M \models \mathscr{L}_{1} \varphi$ is equivalent to $M \models \mathscr{L}_{2} \varphi$ when $\varphi \in \mathscr{L}(\tau)$ and $M$ is a $\tau$-model.

2) For logics $\mathscr{L}_{1}, \mathscr{L}_{2}$ let $\mathscr{L}_{1} \leq \mathscr{L}_{2}$ or $\mathscr{L}_{2}$ is stronger than $\mathscr{L}_{1}$ mean that for every $\tau$ and $\varphi \in \mathscr{L}_{1}(\tau)$ there is $\psi \in \mathscr{L}_{2}(\tau)$ such that $\varphi, \psi$ are equivalent, i.e. $M \models \mathscr{L}_{1} \varphi \Leftrightarrow M \models \mathscr{L}_{2} \psi$ for any $\tau$-model $M$.

3) We say that the logics $\mathscr{L}_{1}, \mathscr{L}_{2}$ are equivalent, $\mathscr{L}_{1} \equiv \mathscr{L}_{2}$ when $\mathscr{L}_{1} \leq \mathscr{L}_{2}$ and $\mathscr{L}_{2} \leq \mathscr{L}_{1}$

Definition 1.20. 1) The logic $\mathbb{L}_{\kappa, \theta}$ for $\kappa \geq \theta \geq \aleph_{0}$ is defined like first order logic, but $\mathbb{L}_{\kappa, \theta}(\tau)$ is the closure of the set of atomic formulas under $\neg \varphi, \bigwedge_{i<\alpha} \varphi_{i}$, where $\alpha<\kappa$ and $\left(\forall x_{0}, \ldots, x_{i}, \ldots\right)_{i<\alpha} \varphi$ where $\alpha<\theta$; a sentence is a formula with no free variables and satisfaction is defined naturally.

1A) First order logic is $\mathbb{L}=\mathbb{L}_{\aleph_{0}, \aleph_{0}}$.

2) Let $\mathbb{L}_{\kappa, \theta, \gamma}(\tau)$ be the set of formulas $\varphi(\bar{x}) \in \mathbb{L}_{\kappa, \theta}(\tau)$ of quantifier depth $\leq \gamma$ and similarly in the other cases.

3) Let $\mathbb{L}_{\leq \kappa, \leq \theta}^{0}=\mathbb{L}_{\kappa^{+}, \theta^{+}}, \mathbb{L}_{<\kappa,<\theta}^{0}=\bigcup\left\{\mathbb{L}_{\leq \kappa_{1}, \leq \theta_{1}}^{0}: \kappa_{1}<\kappa, \theta_{1}<\theta\right\}$; similarly $\mathbb{L}_{\leq \kappa, \leq \theta,<\gamma}^{0}$, etc.

4) Let $\mathbb{L}_{\kappa}^{-1}=\mathbb{L}_{<\kappa, \aleph_{0}}^{0}, \mathbb{L}_{\kappa}^{0}=\mathbb{L}_{<\kappa,<\kappa}^{0}$.

5) We define the logic $\mathbb{L}_{\infty, \theta, \gamma}$ by induction on the ordinal $\gamma$ such that $\mathbb{L}_{\infty, \theta, \gamma}(\tau)$ is a set of cardinality $\leq \beth_{\gamma}\left(|\tau|+\aleph_{0}\right)$, increasing with $\gamma$ as follows:

- for $\gamma=0$, the logic is the set of basic sentences;

- for $\gamma$ limit, it is $\bigcup\left\{\mathbb{L}_{\infty, \theta, \beta}(\tau): \beta<\gamma\right\}$;

- for $\gamma=\beta+1$, it is the set of sentences of the form $(\exists \bar{x}) \bigwedge_{i<\alpha} \varphi_{i}(\bar{x})$ or its negation where $\ell g(\bar{x})<\theta, \varphi_{i}(\bar{x})$ a formula from $\mathbb{L}_{\infty, \theta, \beta}(\tau)$ with $\alpha<$ $\beth_{\beta}\left(|\tau|+\aleph_{0}\right)$.

Observation 1.21. If $\kappa$ is strong limit singular, then there is no logic $\mathscr{L}$ such that:

(a) $\mathbb{L}_{\kappa}^{-1} \leq \mathscr{L} \leq \mathbb{L}_{\kappa}^{0}$

(b) $\mathscr{L}$ satisfies full substitution. 
Note that

Claim 1.22. 1) In Definition 1.20 for $\kappa$ singular, $\mathbb{L}_{\kappa^{+}, \theta} \leq \mathbb{L}_{\kappa, \theta} \subseteq \mathbb{L}_{\kappa^{+}, \theta}$; i.e. every sentence in $\mathbb{L}_{\kappa^{+}, \theta}(\tau)$ is equivalent to one in $\mathbb{L}_{\kappa, \theta}$ (and $\mathbb{L}_{\kappa, \theta}(\tau) \subseteq \mathbb{L}_{\kappa^{+}, \theta}(\tau)$ ), so it seems pointless to allow $\kappa$ to be singular as was originally done. Still this is the tradition and we use it.

2) If $\kappa$ is a regular cardinal, then the logics $\mathbb{L}_{\kappa, \theta}^{0}$ and $\mathbb{L}_{<\kappa, \theta}^{0}$ are equal.

3) If $\kappa=\beth_{\kappa}$, then $\mathbb{L}_{<\kappa,<\kappa,<\kappa}^{0}=\mathbb{L}_{<\kappa,<\kappa}^{0}$.

\section{THE LOGIC $\mathbb{L}_{\kappa}^{1}$}

Our main definition is

Definition 2.1. For a vocabulary $\tau, \tau$-models $M_{1}, M_{2}$, set $\Gamma$ formulas in the vocabulary $\tau$ in any logic (each with finitely many free variables if not said otherwise (see Definition $2.3(4))$, cardinal $\theta$ and ordinal $\alpha$ we define a game $\partial=\partial_{\Gamma, \theta, \alpha}\left[M_{1}, M_{2}\right]$ as follows, and using $\left(M_{1}, \bar{b}_{1}\right),\left(M_{2}, b_{2}\right)$ with their natural meaning when $\operatorname{Dom}\left(\bar{b}_{1}\right)=$ $\operatorname{Dom}\left(\bar{b}_{2}\right)$.

(A) The moves are indexed by $n<\omega$ (but every actual play is finite); just before the $n$-th move we have a state $\mathbf{s}_{n}=\left(A_{n}^{1}, A_{n}^{2}, h_{n}^{1}, h_{n}^{2}, g_{n}, \beta_{n}, n\right)$.

(B) $\mathbf{s}=\left(A^{1}, A^{2}, h^{1}, h^{2}, g, \beta, n\right)=\left(A_{\mathbf{s}}^{1}, A_{\mathbf{s}}^{2}, h_{\mathbf{s}}^{1}, h_{\mathbf{s}}^{2}, g_{\mathbf{s}}, \beta_{\mathbf{s}}, n_{\mathbf{s}}\right)$ is a state (or $n$-state or $(\theta, n)$-state or $(\theta,<\omega)$-state) when:

(a) $A^{\ell} \in\left[M_{\ell}\right] \leq \theta$ for $\ell=1,2$,

(b) $\beta \leq \alpha$, so an ordinal,

(c) $h^{\ell}$ is a function from $A^{\ell}$ into $\omega$,

(d) $g$ is a partial one-to-one function from $M_{1}$ to $M_{2}$ and let $g_{s}^{1}=g^{1}=$ $g_{s}=g$ and let $g_{s}^{2}=g^{2}=\left(g_{s}^{1}\right)^{-1}$,

(e) $\operatorname{Dom}\left(g^{\ell}\right) \subseteq A^{\ell}$ for $\ell=1,2$,

(f) $g$ preserves satisfaction of the formulas in $\Gamma$ and their negation; i.e. for $\varphi(\bar{x}) \in \Gamma$ and $\bar{a} \in^{\ell g(\bar{x})} \operatorname{Dom}(g)$ we have $M_{1} \models \varphi[\bar{a}] \Leftrightarrow M_{2} \models \varphi[g(\bar{a})]$;

$(g)$ if $a \in \operatorname{Dom}\left(g^{\ell}\right)$, then $h^{\ell}(a)<n$.

(C) We define the state $\mathbf{s}=\mathbf{s}_{0}=\mathbf{s}_{\alpha}^{0}$ by letting $n_{\mathbf{s}}=0, A_{\mathbf{s}}^{1}=\emptyset=A_{\mathbf{s}}^{1}, \beta_{\mathbf{s}}=$ $\alpha, h_{\mathbf{s}}^{1}=\emptyset=h_{\mathbf{s}}^{2}, g_{s}=\emptyset$; so really $\mathbf{s}$ depends only on $\alpha$ (but in general, this may not be a state for our game as possibly for some sentence $\psi \in \Gamma$ we have $M_{1} \models \psi \Leftrightarrow M_{2} \models \neg \psi$ ).

(D) We say that a state $\mathbf{t}$ extends a state $\mathbf{s}$ when $A_{\mathbf{s}}^{\ell} \subseteq A_{\mathbf{t}}^{\ell}, h_{\mathbf{s}}^{\ell} \subseteq h_{\mathbf{t}}^{\ell}$ for $\ell=1,2$ and $g_{\mathbf{s}} \subseteq g_{\mathbf{t}}, \beta_{\mathbf{s}}>\beta_{\mathbf{t}}, n_{\mathbf{s}}<n_{\mathbf{t}}$; we say that $\mathbf{t}$ is a successor of $\mathbf{s}$ if in addition $n_{\mathbf{t}}=n_{\mathbf{s}}+1$.

$(E)$ In the $n$-th move,

the anti-isomorphism player (AIS) chooses $\left(\beta_{n+1}, \iota_{n}, A_{n}^{\prime}\right)$ such that:

$\overline{\iota_{n} \in\{1,2\}, \beta_{n+1}<\beta_{n}}$ and $A_{n}^{\iota_{n}} \subseteq A_{n}^{\prime} \in\left[M_{\iota_{n}}\right] \leq \theta$,

the isomorphism player (ISO) chooses a state $\mathbf{s}_{n+1}$ such that

- $\mathbf{s}_{n+1}$ is a successor of $\mathbf{s}_{n}$

- $A_{\mathbf{s}_{n+1}}^{\iota_{n}}=A_{n}^{\prime}$

- $A_{\mathbf{s}_{n+1}}^{3-\iota_{n}}=A_{\mathbf{s}_{n}}^{3-\iota_{n}} \cup \operatorname{Dom}\left(g_{\mathbf{s}_{n+1}}^{3-\iota_{n}}\right)$

- if $a \in A_{n}^{\prime} \backslash A_{\mathbf{s}_{n}}^{\iota_{n}}$, then $h_{\mathbf{s}_{n+1}}^{\iota_{n}}(a) \geq n+1$ 
- $\operatorname{Dom}\left(g_{\mathbf{s}_{n+1}}^{\iota_{n}}\right)=\left\{a \in A_{\mathbf{s}_{n}}^{\iota_{n}}: h_{\mathbf{s}_{n}}^{\iota_{n}}(a)<n+1\right\}$, so it includes $\operatorname{Dom}\left(g_{\mathbf{s}_{n}}^{\iota_{n}}\right)$

- $\beta_{\mathbf{s}_{n+1}}=\beta_{n+1}$.

$(F) \bullet \quad$ The play ends when one of the player has no legal moves (always occur as $\left.\beta_{n}<\beta_{n-1}\right)$ and then this player loses; this may occur for $n=0$.

- $\quad$ For $\alpha=0$ we stipulate that ISO wins iff $\mathbf{s}_{\alpha}^{0}$ is a state.

Discussion 2.2. 1) This is a parallel to EF-games. Note that we like on the one hand for the game to have $\leq \omega$ moves, really each play has $<\omega$ moves and deals with sets of cardinality $\leq \theta$ and on the other hand we do not like well ordering to be definable, i.e. allow $M_{1}$ to be well ordered while $M_{2}$ to be non-well ordered but still the ISO player wins. We do this by "rescheduling our debts", i.e. using the $h_{n}$ 's.

Definition 2.3. 1) Let $\mathscr{E}_{\Gamma, \theta, \alpha}^{0, \tau}$ be the class $\left\{\left(M_{1}, M_{2}\right): M_{1}, M_{2}\right.$ are $\tau$-models and in the game $\partial_{\Gamma, \theta, \alpha}\left[M_{1}, M_{2}\right]$ the ISO player has a winning strategy $\}$, where $\Gamma$ is a set of formulas in the vocabulary $\tau$, each with finitely many free variables.

2) $\mathscr{E}_{\Gamma, \theta, \alpha}^{1, \tau}$ is the closure of $\mathscr{E}_{\Gamma, \theta, \alpha}^{0, \tau}$ to an equivalence relation (on the class of $\tau$ models).

3) Above we may replace $\Gamma$ by $\mathrm{qf}(\tau)$, which means that $\Gamma=$ the set at $(\tau)$ or $\operatorname{bs}(\tau)$ formulas in the vocabulary $\tau$.

4) Above if we omit $\tau$ we mean that $\tau=\tau_{\Gamma}$ and if we omit $\Gamma$ we mean $\operatorname{bs}(\tau)$. Abusing notation we may say that $M_{1}, M_{2}$ are $\mathscr{E}_{\Gamma, \theta, \alpha}^{0, \tau}$-equivalent.

Fact 2.4. Assume $\partial_{\Gamma, \theta, \alpha}\left[M_{1}, M_{2}\right]$ is well defined and $M_{1}, M_{2}$ are $\tau$-models.

1) The game $\partial_{\Gamma, \theta, \alpha}\left[M_{1}, M_{2}\right]$ is a determined game and is without memory; i.e. during a play, being a winning situation does not depend on the history, just on the current state, also only $M_{\ell}\left\lceil\tau_{\Gamma}\right.$ are relevant.

2a) The relation $\mathscr{E}_{\Gamma, \theta, \alpha}^{0}$ holds for $\left(M_{1}, M_{2}\right)$ when $M_{1}, M_{2}$ are isomorphic $\tau$-models.

2b) If $M_{1} \cong M_{1}^{\prime}, M_{2} \cong M_{2}^{\prime}$, then $M_{1} \mathscr{E}_{\Gamma, \theta, \alpha}^{0} M_{2} \Leftrightarrow M_{1}^{\prime} \mathscr{E}_{\Gamma, \theta, \alpha}^{0} M_{2}^{\prime}$.

2c) $\mathscr{E}_{\Gamma, \theta, \alpha}^{0}$ is reflexive and symmetric.

3) The relation $\mathscr{E}_{\Gamma, \theta, \alpha}^{1}$ is an equivalence relation on the class of $\tau$-models.

4) If $\alpha$ is a limit ordinal, then $M_{1} \mathscr{E}_{\Gamma, \theta, \alpha}^{0} M_{2}$ iff $\left[\beta<\alpha \Rightarrow M_{1} \mathscr{E}_{\Gamma, \theta, \beta}^{0} M_{2}\right]$.

5) $\mathscr{E}_{\Gamma, \theta, \alpha}^{1}$ has $\leq \beth_{\alpha+1}(|\Gamma|+\theta)$ equivalence classes.

6) If $\tau^{1} \subseteq \tau^{2}, \Gamma_{1} \subseteq \Gamma_{2}, \theta_{1} \leq \theta_{2}$ and $\alpha_{1} \leq \alpha_{2}$ and $M_{1} \mathscr{E}_{\Gamma_{2}, \theta_{2}, \alpha_{2}}^{0, \tau^{2}} M_{2}$, then $M_{1} \mathscr{E}_{\Gamma_{1}, \theta_{1}, \alpha_{1}}^{0, \tau^{1}} M_{2}$.

Proof. 1) Obvious.

2a) Let $g_{*}$ be an isomorphism from $M_{1}$ onto $M_{2}$. Now a winning strategy for the player ISO in $\partial_{\Gamma, \theta, \alpha}\left[M_{1}, M_{2}\right]$ is to preserve " $g_{\mathbf{s}_{n}} \subseteq g_{*}$ ".

2b) Should be clear.

2c) Let us check.

Reflexivity:

Follows from part (2a) as $M \cong M$.

Symmetry:

Reading the definition carefully it should be clear.

3),4) Easy, too.

5) We prove by induction on $\alpha$ that there is an equivalence relation $\mathscr{E}_{\Gamma, \theta, \alpha}^{2}$ on the class of $\tau(\Gamma)$-models such that $M_{1} \mathscr{E}_{\Gamma, \theta, \alpha}^{2} M_{2} \Rightarrow M_{1} \mathscr{E}_{\Gamma, \theta, \alpha}^{0} M_{2}$ and $\mathscr{E}_{\Gamma, \theta, \alpha}^{2}$ has $\leq \beth_{\alpha+1}(|\Gamma|+\theta)$ equivalence classes. For $\alpha=0$, recall clause $(\mathrm{F})$ of Definition 2.1 . 
so easily $M_{1} \mathscr{E}_{\Gamma, \theta, \alpha}^{0} M_{2}$ means that $\Gamma_{\ell}=\left\{\varphi: M_{\ell} \models \varphi\right.$ and $\varphi \in \Gamma, \varphi$ is a sentence $\}$ does not depend on $\ell$; clearly $\mathscr{E}_{\Gamma, \theta, \alpha}^{0}$ is an equivalence relation with $\leq 2^{|\Gamma|}$ equivalence classes and $\mathscr{E}_{\Gamma, \theta, \alpha}^{1}=\mathscr{E}_{\Gamma, \theta, \alpha}^{0} ;$ let $\mathscr{E}_{\Gamma, \theta, \alpha}^{2}=\mathscr{E}_{\Gamma, \theta, \alpha}^{0}$. For $\alpha$ a limit ordinal use part (4) and choose $\mathscr{E}_{\Gamma, \theta, \alpha}^{2}=\bigcap\left\{\mathscr{E}_{\Gamma, \theta, \beta}^{2}: \beta<\alpha\right\}$, recalling that $\beth_{\alpha}(|\Gamma|+\theta)=\sum\left\{\beth_{\beta}(|\Gamma|+\theta)\right.$ : $\beta<\alpha\}$.

Lastly, for $\alpha=\beta+1$ use the induction hypothesis and $\beth_{\alpha}(|\Gamma|+\theta)=2^{\beth_{\beta}(|\Gamma|+\theta)}$. Alternatively use $\mathbb{L}_{\theta^{+}, \theta^{+}, \alpha}$; i.e. use the proof of Claim 2.8 , when we replace $\beth_{\alpha}(|\Gamma|+$

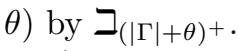

6) Easy.

Definition 2.5. We define the logic $\mathbb{L}_{\leq \theta}^{1}$ as follows: a sentence $\psi \in \mathbb{L}_{\leq \theta}(\tau)$ iff the sentence is defined using (or by) a triple ( $\left.\mathrm{qf}\left(\tau_{1}\right), \theta, \alpha\right)$ which means: $\tau_{1}$ is a sub-vocabulary of $\tau$ of cardinality $\leq \theta$ and $\alpha<\theta^{+}$and for some sequence $\left\langle M_{\alpha}\right.$ : $\alpha<\alpha(*)\rangle$ of $\tau_{1}$-models of length $\alpha(*) \leq \beth_{\alpha+1}(\theta)$ we have: $M \models \psi$ iff $M$ is $\mathscr{E}_{\mathrm{qf}\left(\tau_{1}\right), \theta, \alpha}^{1}$-equivalent to $M_{\alpha}$ for some $\alpha<\alpha(*)$.

2) Let $\mathbb{L}_{\kappa}^{1}=\bigcup\left\{\mathbb{L}_{\leq \theta}^{1}: \theta<\kappa\right\}$, so $\mathbb{L}_{\theta^{+}}^{1}=\mathbb{L}_{\leq \theta}^{1}$.

Remark 2.6. 1) The present definition of the logic in Definition 2.5 is interesting mainly for $\kappa$ a strong limit such that $\kappa=\beth_{\kappa}$ and it seems to me that it makes the presentation transparent. Note that $\mathbb{L}_{\leq \theta}^{1}$ is similar to the set of formulas of quantifier depth $<\theta^{+}$.

2) Why? Just note that for vocabulary $\tau$ of cardinality $\leq \theta, \mathbb{L}_{\leq \theta}^{1}(\tau)$ has cardinality $\beth_{\theta^{+}}$, and it helps to have arbitrary Boolean combinations of formulas of a fixed quantifier depth $\left[<\theta^{+}\right]$. Note that if $\varphi(\bar{x}) \in \mathbb{L}_{\kappa}^{1}(\tau)$ has infinitely many free variables, we cannot "close" it to a sentence.

3) We may instead define $\mathbb{L}_{\leq \theta}^{1, *}$ by: $\psi \in \mathbb{L}_{\leq \theta}^{1}(\tau)$ iff for some $\tau^{\prime} \subseteq \tau$ of cardinality $\leq \theta$ and some sequence $\left\langle M_{i}: i<i(*)\right\rangle$ of $\tau^{\prime}$-models of cardinality $\leq 2^{\theta}$ and some $\gamma<\theta^{+}$we have: a $\tau$-model $M$ satisfies $\psi$ iff $M \mathscr{E}_{\mathrm{qf}\left(\tau^{\prime}\right), \theta, \gamma}^{1} M_{i}$ for some $i$.

Claim 2.7. 1) $\mathbb{L}_{\leq \theta}^{1}$ is a nice logic; see Definition 1.9

2) The logic $\mathbb{L}_{\leq \theta}^{1}$ has full substitution.

3) $\mathbb{L}_{\leq \theta}^{1}(\tau)$ has cardinality $\leq|\tau|^{\theta}+\beth_{\theta^{+}}$for any vocabulary $\tau$.

4) If $\kappa=\beth_{\kappa}$, then $\mathbb{L}_{\kappa}^{1}$ is a nice logic, $\mathbb{L}_{\kappa}^{1}(\tau)$ has cardinality $\kappa$ whenever $\tau$ is a vocabulary of cardinality $<\kappa$ and $\mathbb{L}_{\kappa}^{1}(\tau)$ has cardinality $|\tau|^{<\kappa}+\kappa$ for any vocabulary $\tau$.

5) In part (4), if $\kappa$ is regular, then the logic $\mathbb{L}_{\kappa}^{1}$ has full substitution.

6) If $\theta, \alpha<\kappa$ and $\tau$ is a vocabulary of cardinality $<\kappa$, then for any set $\mathscr{U}$ of $\mathscr{E}_{\mathrm{qf}(\tau), \theta, \alpha}$-equivalence classes for some $\psi \in \mathbb{L}_{\kappa}^{1}(\tau)$ we have $\left\{M: M / \mathscr{E}_{\mathrm{qf}(\tau), \theta, \alpha}^{1} \in\right.$ $\mathscr{U}\}=\{M: M$ a $\tau$-model of $\psi\}$.

Proof. 1), 2) Just check Definitions 1.9, 1.12, 1.16(5) but still elaborate upon the relatively more substantial Definitions 1.12, 1.16(5).

Clause $(a)$ : Atomic formulas

Just note that if $M_{1} \mathscr{E}_{\mathrm{qf}(\tau), \theta, \alpha} M_{2}$ for $\alpha=0$ (any $\theta$ ), then $M_{1}, M_{2}$ satisfies the same $\tau$-atomic sentences. 
Clause $(b)(\alpha)$ : Conjunction, (similarly Disjunction)

Just note that if $\varphi_{\ell}$ is defined using $\left(\mathrm{qf}\left(\tau_{\ell}\right), \theta_{\ell}, \alpha_{\ell}\right)$ and if $\tau=\tau_{1} \cup \tau_{2}, \theta=$ $\max \left\{\theta_{1}, \theta_{2}\right\}, \alpha=\max \left\{\alpha_{1}, \alpha_{2}\right\}$, then the equivalence relation $\mathscr{E}_{\mathrm{qf}(\tau), \theta, \alpha}^{1}$ refines the equivalence relation $\mathscr{E}_{\mathrm{qf}\left(\tau_{\ell}\right), \theta_{\ell}, \alpha_{\ell}}$ for $\ell=1,2$.

Clause $(b)(\beta)$ : Existential Quantifier

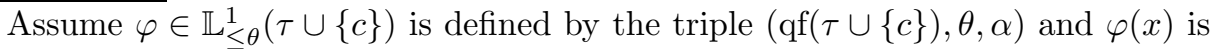
the corresponding formula $\in \mathbb{L}_{\leq \theta}^{1}(\tau)$ and $\exists x \varphi(x)$ is the naturally defined sentence.

Now if $M_{1}, M_{2}$ are $\mathscr{E}_{\mathrm{qf}(\tau), \theta, \alpha+1}^{0}$-equivalent $\tau$-models, then $M_{1} \models \exists x \varphi(x)$ iff $M_{2} \models$ $(\exists x) \varphi(x)$ by the definition of the game. Hence this holds for " $M_{1}, M_{2}$ are $\mathscr{E}_{\mathrm{qf}(\tau), \theta, \alpha+1}^{1}$ equivalent $\tau$-models".

Clause $(b)(\gamma)$ : Negation

Obvious by the definition because for any vocabulary $\tau$ of cardinality $\leq \theta$ the equivalence relation $\mathscr{E}_{\mathrm{qf}(\tau), \theta, \alpha}^{1}$ has $\leq \beth_{\alpha+1}(\theta)$ equivalence classes by Fact $2.4(5)$.

Clause $(c)$ : Restricting to a unary predicate $P$

Easily follows if $\psi$ is defined using ( $\mathrm{qf}(\tau), \theta, \alpha)$ and without loss of generality $P \in \tau$. Then so is $\psi\lceil P$.

Clause $(d)$ : We prove more: Full substitution:

Assume we are given vocabularies $\tau_{1}, \tau_{2}$ and consider substituting $\varphi_{P}\left(x_{0}, \ldots\right.$, $\left.x_{\operatorname{arity}_{\tau_{2}}(P)-1}\right) \in \mathbb{L}_{\leq \theta}^{1}\left(\tau_{0}\right)$ for $P \in \tau_{2}$ treating $F\left(x_{0}, \ldots, x_{\operatorname{arity}(F)-1}\right)=x_{\operatorname{arity}(F)}$ as an $\left(\operatorname{arity}_{\tau_{2}(F)+1}\right)$-place predicate. Let $\varphi_{P}$ be defined by $\left(\mathrm{qf}\left(\tau^{+\operatorname{arity}(P)}\right), \theta_{P}, \alpha_{P}\right)$ and let $\alpha_{0}=\sup \left\{\alpha_{P}: P \in \tau_{2}\right\}$.

We are given $\psi \in \mathbb{L}_{\leq \theta}^{1}\left(\tau_{1}\right)$ and we shall find $\psi^{\prime} \in \mathbb{L}_{\leq \theta}^{1}\left(\tau_{2}\right)$, which says that if we substitute $\varphi_{P}\left(x_{0}, \ldots, x_{\operatorname{arity}_{\tau_{2}}(P)}\right)$ instead of $P\left(x_{0}, \ldots\right)$ in $\psi$ for every $P \in \tau_{1}$, we get (up to equivalence) $\psi^{\prime}$. Let $\psi$ be defined by $\left(\mathrm{qf}\left(\tau_{1}\right), \theta_{1}, \alpha_{1}\right)$. Let $\alpha_{2}=\alpha_{1}+\alpha_{0}$ and then easily there is $\psi^{\prime}$ as required defined by $\left(\mathrm{qf}\left(\tau_{2}\right), \theta_{1}, \alpha_{2}\right)$.

2.7

Claim 2.8. 1) Let $\kappa=\beth_{\theta^{+}}$.

$\mathbb{L}_{\leq \theta}^{1} \leq \mathbb{L}_{\kappa}^{0}$; i.e. every formula of $\mathbb{L}_{\leq \theta}^{1}$ is equivalent to, hence can be looked at, as a formula of $\mathbb{L}_{\kappa}^{0}$.

2) $\mathbb{L}_{\leq \theta}^{-1} \leq \mathbb{L}_{\leq \theta}^{1}$.

Remark 2.9. For many purposes we identify them, i.e. say $\mathbb{L}_{\leq \theta}^{1} \subseteq \mathbb{L}_{\kappa}^{0}$; this gives another reasonable version of subformulas.

Proof. 1) We first prove:

$\boxplus_{1}$ If $\mathbf{s}$ is a state in the game $\partial_{\mathrm{qf}(\tau), \theta, \alpha}\left[M_{1}, M_{2}\right]$ and $\beta=\beta_{\mathbf{s}}, \tau=\tau\left(M_{\ell}\right), \bar{a}_{1}=$ $\left\langle a_{\varepsilon}^{1}: \varepsilon<\varepsilon(*)\right\rangle \in \theta^{+}>\left(M_{1}\right)$, then list $\operatorname{Dom}\left(g_{\mathbf{s}}\right)$ and $\bar{a}_{2}=\left\langle a_{\varepsilon}^{2}: \varepsilon<\varepsilon(*)\right\rangle$, where $a_{\varepsilon}^{2}=g_{\mathbf{s}}\left(a_{\varepsilon}^{1}\right)$ and $M_{2} \models \varphi\left[\bar{a}_{1}\right] \Leftrightarrow M_{2} \models \varphi\left[\bar{a}_{2}\right]$ for every $\varphi=\varphi\left(\left\langle x_{\varepsilon}\right.\right.$ : $\varepsilon<\varepsilon(*)\rangle) \in L_{\beta}^{*}:=\bigcup_{\zeta<\beta} \mathbb{L}_{\left(\beth_{\zeta}(\theta+|\tau|)\right)^{+}, \theta^{+}}(\tau)$, or just $\varphi \in L_{\beta}^{*}=\mathbb{L}_{\infty, \theta^{+}, \beta}(\tau)$; see Definition [1.20 $(5)$, then $\mathbf{s}$ is a winning state for the player ISO in the game.

[Why? We prove this by induction on $\beta$. First, for $\beta=0$ this is trivial as $\mathbf{s}$ is a state. Second, for $\beta$ limit, any choice of the AIS player includes an ordinal $\gamma=\beta_{n_{\mathbf{s}}+1}<\beta$, so the ISO player may "pretend" that the given state $\mathbf{s}$ has $\beta_{\mathbf{s}}=\gamma+1$ and use the induction hypothesis. Third, if $\beta=\gamma+1$, let the AIS player make his choice $\left(\beta_{n_{\mathbf{s}}+1}, \iota, A\right)$. Now ISO has to extend $g_{\mathbf{s}}^{\iota}$ adding some $\leq \theta$ elements of $M_{\iota}$ to its domain, the elements in $\left\{a \in \operatorname{Dom}\left(g_{\mathbf{s}}^{\iota}\right): h_{\mathbf{s}}^{\iota}(a)<n_{\mathbf{s}}+1\right\} \cup A$. 
Let $\bar{b}_{\iota}$ list them. Let $\bar{x}=\left\langle x_{\varepsilon}: \varepsilon<\varepsilon(*)\right\rangle$ and let $\bar{y}=\left\langle y_{\varepsilon}: \varepsilon<\ell g\left(\bar{b}_{\iota}\right)\right\rangle$ and define $\varphi_{*}(\bar{y}, \bar{x})=\bigwedge\left\{\varphi(\bar{y}, \bar{x}) \in L_{\gamma}^{*}: M_{\iota} \models \varphi\left[\bar{b}, \bar{a}_{\iota}\right]\right\}$.

So $M_{\iota} \models \varphi_{*}\left[\bar{b}, \bar{a}_{\iota}\right]$; hence $M_{\iota} \models(\exists \bar{y}) \varphi_{*}\left(\bar{y}, \bar{a}_{\iota}\right)$ and hence by the assumption of $\boxplus_{1}$ we have $M_{3-\iota} \models$ " $(\exists \bar{y}) \varphi_{*}\left(\bar{y}, \bar{a}_{3-\iota}\right)$ ". Hence there is $\bar{b}_{3-\iota} \in{ }^{\ell g\left(\bar{b}_{\iota}\right)}\left(M_{3-\iota}\right)$ such that $M_{3-\iota}=\varphi_{*}\left[\bar{b}_{3-\iota}, \bar{a}_{3-\iota}\right]$.

Now the player ISO can make its move getting the state $\mathbf{t}$, a successor of $\mathbf{s}$ such that $g_{\mathbf{t}}=g_{\mathbf{s}} \cup\left\{\left\langle b_{\varepsilon}^{1}, b_{\varepsilon}^{2}\right\rangle: \varepsilon<\ell g(\bar{y})\right\rangle, g_{\mathbf{t}}$ maps $\bar{b}_{1}$ to $\bar{b}_{2}$ and $n_{\mathbf{t}}=n_{\mathbf{s}}+1$ and $\beta_{\mathbf{t}}=\gamma$, clearly possible. As the ordinal $\beta_{n_{\mathbf{s}}+1}$ chosen by the AIS is $\leq \gamma$ also $\mathbf{t}$ is as required in $\boxplus_{1}$ but so, by the induction hypothesis also it is a winning state.]

Let

$\boxplus_{2} \mathscr{E}_{\tau, \theta, \alpha}=\left\{\left(M_{1}, M_{2}\right): M_{1}, M_{2}\right.$ are $\tau$-models satisfying the same sentences from $\left.L_{\alpha}^{*}\right\}$.

Now we shall prove $\boxplus_{3}$ and by $\boxplus_{3}(d)$ we are done.

$\boxplus_{3}(a) \quad \mathscr{E}_{\tau, \theta, \alpha}$ is an equivalence relation with $\leq\left(\beth_{\alpha+1}(\theta+|\tau|)\right)$ equivalence classes.

(b) $\mathscr{E}_{\tau, \theta, \alpha}$ is an equivalence relation included in $\left\{\left(M_{1}, M_{2}\right): M_{1}, M_{2}\right.$ are $\tau$-models such that the player ISO wins in $\left.\partial_{\mathrm{qf}(\tau), \theta, \alpha}\left[M_{1}, M_{2}\right]\right\}$.

(c) $\mathscr{E}_{\tau, \alpha, \theta}$ refines the equivalence relation $\mathscr{E}_{\mathrm{qf}(\tau), \theta, \alpha}^{1}$.

(d) $\quad \mathscr{E}_{\mathrm{qf}(\tau), \theta, \alpha}^{1}$ has $\leq \beth_{\alpha+1}(\theta+|\tau|)$-equivalence classes.

[Why? Clause (a) follows from the number of such sentences being $\leq \beth_{\alpha}(\theta+|\tau|)$; clause (b) follows by $\boxplus_{1}$; clause (c) follows from (b) and the definitions of $\mathscr{E}_{\mathrm{qf}(\tau), \theta, \alpha}^{0}$ and $\mathscr{E}_{\mathrm{qf}(\tau), \theta, \alpha}$. Lastly, clause (d) follows from clauses (a) + (c).

2) Easy.

Remark 2.10. 1) The proof above may seem wasteful but for our purposes this is immaterial.

2) We can do better as follows; i.e., another proof of Claim 2.8 runs as follows:

By induction on the formula. Without loss of generality we deal with a formula $\Theta_{N, \bar{d}, \theta, \alpha, \Gamma}(\bar{x})$; by induction on $\beta \leq \alpha$ we prove:

$\boxplus_{1}$ If $n \in[-1, \omega)$ and $\gamma(1), \gamma(2)<\theta^{+}, \bar{b} \in \gamma(2) N, \mathbf{h}_{\ell}: \gamma(\ell) \rightarrow \omega$ letting $\bar{x}=$ $\left\langle x_{i}: i<\gamma(2)\right\rangle ; u_{\ell} \subseteq \gamma(\ell)$ for $\ell=1,2$, g a one-to-one mapping from $u_{1}$ onto $u_{2}$ (so $\left.n=-1 \Rightarrow \gamma(1), \gamma(2)=0\right)$, then for some formula $\varphi(\bar{z}, \bar{x})=$ $\varphi_{N, \bar{d}, \theta, \alpha, \Gamma, \mathbf{h}_{1}, \mathbf{h}_{2}, g, \beta}^{0}(\bar{x}) \in \mathbb{L}_{\kappa}^{0}$ we have: for any model $M$ of the vocabulary of $N$ and $\bar{c} \in{ }^{(\ell g(\bar{d}))} M$ and $\bar{a} \in{ }^{\ell g(\bar{b})} M$, letting

- $A_{n}^{1}=\operatorname{Rang}(\bar{c}), A_{n}^{2}=\operatorname{Rang}(\bar{d})$,

- $g_{n}$ has domain $\left\{c_{i}: i \in u\right\}$ and $g_{n}\left(c_{i}\right)=d_{\mathbf{g}(i)}$ for $i \in u$,

- $h_{n}^{\ell}\left(c_{i}\right)=d_{\mathbf{h}_{\ell}}(i)$,

the following are equivalent:

(i) $M \models \varphi[\bar{c}, \bar{a}]$,

(ii) $\left(A_{n}, B_{n}, h_{n}^{1}, h_{n}^{2}, g_{n}, \beta\right)$ is a winning position for the equivalence player in the game $\partial_{N, \theta, \alpha, \Gamma}[(M, \bar{c}),(N, \bar{d})]$. 
$\boxplus_{2}$ Similarly we have a formula $\varphi_{N, \bar{c}, \theta, \alpha, \Gamma, h, h^{\prime}, \beta}^{1}(\bar{z}, \bar{x})$ expressing the situation after the AIS player moves.

The proof is straightforward.

The Crucial Claim 2.11. We have $M_{n} \equiv_{\mathbb{L}_{\theta}^{1}} M_{\omega}$ for $n<\omega$ and even $M_{n} \models \psi[\bar{a}] \Leftrightarrow$ $M_{\omega} \models \psi[\bar{a}]$ when:

(a) $\psi(\bar{z}) \in \mathbb{L}_{\leq \theta}^{1}(\tau)$, a formula,

(b) $M_{n} \prec_{\mathbb{L}_{\partial^{+}, \theta^{+}}} M_{n+1}$, where $\partial=\beth_{\theta^{+}}$,

(c) $M_{\omega}:=\bigcup_{n<\omega} M_{n}$,

(d) $\bar{a} \in{ }^{\ell g(\bar{z})}\left(M_{0}\right)$,

(e) $\tau=\tau\left(M_{n}\right)$ for $n<\omega$.

Remark 2.12. In fact the logic $\mathbb{L}_{\partial^{+}, \theta^{+}, \theta^{+}}^{0}$ suffices; see Definition 1.20(2).

Proof. Without loss of generality, $|\tau| \leq \theta$. We shall prove by induction on $\beta<\theta^{+}$ the following:

$\boxplus_{\beta} \mathbf{s}$ is a winning state for the ISO player in the game $\partial_{\mathrm{qf}(\tau), \theta, \alpha}\left[M_{n}, M_{\omega}\right] \underline{\text { when }}$ for some $k$ we have

(a) $\mathbf{s}$ is a state in this game,

(b) $n \leq k<\omega$,

(c) $\beta_{\mathbf{s}}=\beta$,

(d) $\operatorname{Rang}\left(g_{\mathbf{s}}^{2}\right) \subseteq M_{k}$,

(e) if $b \in A_{\mathbf{s}}^{2}$, then $b \in M_{h_{\mathbf{s}}^{2}(b)}$,

(f) letting $\bar{a}^{1}$ list $\operatorname{Dom}\left(g_{\mathbf{s}}\right)$ and $\bar{a}^{2}$ be $\left\langle g_{\mathbf{s}}\left(a_{i}^{1}\right): i<\ell g\left(\bar{a}^{1}\right)\right\rangle$ and $\lambda_{\beta}=\beth_{\beta}(\theta)$ for every $\varphi(\bar{x}) \in \mathbb{L}_{\lambda_{\beta}^{+}, \theta^{+}}$we have $M_{k} \models \varphi\left[\bar{a}^{1}\right] \Leftrightarrow M_{\omega} \models \varphi\left[\bar{a}^{2}\right]$.

The case $\mathbf{s}=\mathbf{s}_{\beta}^{0}$, the initial state, suffices to prove the desired result.

First Case: $\beta=0$.

Trivial.

Second Case: $\beta$ a limit ordinal.

If the AIS makes its move choosing $\left(A, \iota, \beta_{1}\right)$, the ISO player may pretend that $\beta_{\mathbf{s}}=\beta+1$ and use the induction hypothesis. This is O.K. as $\beta<\beta+1$, as in proving Claim 2.8 .

Third Case: $\beta=\gamma+1$.

As in the proof of Claim 2.8

We can sum up the easy properties but first we present two definitions.

Definition 2.13. 1) We say that $N$ codes $[N]^{\leq \lambda}$ when for some two-place predicate $R$ we have that $\{\operatorname{set}(b, N): b \in N\}$ is a cofinal subset of $([N] \leq \lambda, \subseteq)$.

2) In this case for $b \in N$ let $\operatorname{set}(b, N)=\operatorname{set}_{R}(b, N)=\left\{a: a R^{N} b\right\}$ and if $R$ is clear from the context we may omit it.

For our results it seems helpful to define some variants of " $\mathscr{L}$ satisfies the downward LST". 
Definition 2.14. Let $\mathscr{L}$ be a logic, $\tau_{0}$ a vocabulary, $\psi_{0} \in \mathscr{L}\left(\tau_{0}\right)$ a sentence with $\tau_{0}$ of minimal cardinality or $T \subseteq \mathscr{L}\left(\tau_{0}\right)$, i.e. a set of sentences with $\left|\tau_{0}\right|$ minimal.

1) $\mathscr{L}$ satisfies $\operatorname{LST}_{<\kappa}^{\iota}$ when for every vocabulary $\tau$ of cardinality $<\kappa$ and $\varphi \in$ $\mathscr{L}(\tau)$ we have $\operatorname{LST}_{<\kappa}^{\iota}(\varphi)$; see below. If $\iota=0$, we may omit it.

If $\kappa=\lambda^{+}$, we may write " $\lambda$ " instead " $<\kappa$ ". $\operatorname{LST}_{<\kappa}^{\iota}(\psi)$ means $\operatorname{LST}_{<\kappa}^{\iota}(\{\psi\})$ and similarly below and $\mathrm{LST}_{<\kappa}^{\iota}(\leq \theta)$ means $\operatorname{LST}_{<\kappa}^{\iota}(T)$ when $|T| \leq \theta$; the reader may concentrate on the case $\kappa=\lambda^{+}$.

2) For $\iota=0$, let $\operatorname{LST}_{<\kappa}^{\iota}(T)$ mean: if $T$ has a model $N$ of cardinality $\geq \kappa$, then it has a model $M$ of cardinality $\lambda$ for arbitrarily large cardinals $\lambda<\kappa$.

3) If $\iota=1$, similarly but $M \subseteq N$ (moreover we can demand $A \subseteq M$ for a given $\left.A \in[N]^{<\kappa}\right)$.

4) For $\iota=2$, let $\operatorname{LST}_{<\kappa}^{\iota}(T)$ mean: if $N$ is a model of $T$ of cardinality $\geq \kappa$, then for arbitrarily large $\lambda<\kappa$ we have that if $N^{+}$is an expansion of $N$ and $\tau\left(N^{+}\right)$has cardinality $\leq \lambda$, then some $M^{+} \subseteq N^{+}$of cardinality $\lambda$ satisfying $M=M^{+} \mid \tau_{T}$ is a model of $T$.

5) For $\iota=3$, as in $\iota=2$, but we further assume that $N^{+}$codes $[N]^{\leq \lambda}$; see Definition 2.13 and we further conclude that for some $\left\langle d_{n}: n<\omega\right\rangle \in{ }^{\omega}\left(M^{+}\right)$we have $\left|M^{+}\right|=\bigcup\left\{\operatorname{set}\left(d_{n}, N^{+}\right): n<\omega\right\}$.

6) We define $\operatorname{LST}_{<\kappa}^{\iota, *}(T)$ similarly but for a pregiven $\mu<\kappa$ we can demand that $\|M\|=\|M\|^{\mu}$ and similarly $\operatorname{LST}_{<\kappa}^{L, *}$.

Conclusion 2.15. Assume $\theta<\kappa=\beth_{\kappa}$.

1) $\mathbb{L}_{\leq \theta}^{1}$ is a nice logic with full substitution; see Definition 1.12 ,

2) $\mathbb{L}_{\kappa}^{1}$ is a nice logic and if $\kappa$ is regular (equivalently strongly inaccessible), then it has full substitution.

3) $\mathbb{L}_{\kappa}^{-1} \leq \mathbb{L}_{\kappa}^{1} \leq \mathbb{L}_{\kappa}^{0}$; in fact, $\mathbb{L}_{\theta^{+}, \aleph_{0}} \leq \mathbb{L}_{\leq \theta}^{1} \leq \mathbb{L}_{\beth_{\theta^{+}}, \theta^{+}}$(really $\mathbb{L}_{\leq \theta}^{1} \leq \mathbb{L}_{\beth_{\theta^{+}}, \theta^{+}, \theta^{+}}$ and even $\leq \mathbb{L}_{\infty, \theta^{+}, \theta^{+}}$).

4) $\mathbb{L}_{\kappa}^{1}$ satisfies the following versions of the downward LST:

(a) Every sentence $\psi \in \mathbb{L}_{\kappa}^{1}$ which has a model has a model of cardinality $<\kappa$.

(b) $\mathbb{L}_{\kappa}^{1}$ has $\mathrm{LST}_{<\kappa}^{\iota}$ for $\iota \leq 3$ (see Definition 2.14 above).

(c) For every $\psi \in \mathbb{L}_{\kappa}^{1}$ there is $\partial<\kappa$ such that: if $N$ is a model of $\psi$ of cardinality $\lambda$ and $\mu=\mu^{<\partial} \leq \lambda$ or at least $\mu=\sum_{n<\omega} \mu_{n} \leq \lambda$ and $\left(\mu_{n}\right)^{<\partial}=\mu_{n}$ for $n<\omega$, then some submodel $M$ of $N$ of cardinality $\mu$ is a model of $\psi$.

Proof. 1) By Claim 2.7 .

2) Follows as $\mathbb{L}_{\leq \theta}^{1}$ is $\subseteq$-increasing with $\theta$ and the defintion of $\mathbb{L}_{\kappa}^{1}$ in Definition 2.5(2).

3) We have $\mathbb{L}_{\leq \theta}^{1} \leq \mathbb{L}_{\beth_{\theta^{+}}, \theta^{+}}$(and moreover $\mathbb{L}_{\leq \theta}^{1} \leq \mathbb{L}_{\beth_{\theta^{+}}, \theta^{+}, \theta^{+}}$) by Claim 2.8, So by the definition, $\mathbb{L}_{\kappa}^{1} \leq \mathbb{L}_{\kappa}^{0}$. Note that if $M_{1}, M_{2}$ are $\mathscr{E}_{\mathrm{qf}(\tau), \aleph_{0}, \alpha}$-equivalent, then $M_{1} \models \psi \Leftrightarrow M_{2} \models \psi$ for every $\psi \in \mathbb{L}_{\beth_{\alpha}^{+}, \aleph_{0}, \alpha}$; hence $\mathbb{L}_{\theta^{+}, \aleph_{0}} \leq \mathbb{L}_{\leq \theta}^{1}$ and so by the definitions, $\mathbb{L}_{\kappa}^{-1} \leq \mathbb{L}_{\kappa}^{1}$.

4) Clause (a) follows by part (3) and the parallel results on $\mathbb{L}_{\lambda^{+}, \theta^{+}}$of Hanf (see, e.g., [Dic85]). Clauses (b), (c) follow by Claim 2.11.

\section{Serious properties of $\mathbb{L}_{\kappa}^{1}$}

First we prove a strong form of non-definability of well ordering.

Claim 3.1. Let $\kappa=\beth_{\kappa}$, so a strong limit cardinal. 
1) Property (d) of Problem 1.5 holds.

2) Moreover, if (A), then (B), where $\tau$ is a vocabulary to which the predicates $P,<, R$ (unary, binary, binary) belong and:

(A) (a) $\psi \in \mathbb{L}_{\leq \theta}^{1}(\tau)$ is defined using $(\mathrm{qf}(\tau), \theta, \alpha)$,

(b) $\theta \geq|\tau|$,

(c) $\partial=\beth_{\alpha+1}(\theta)$,

(d) $\mu=2^{\partial}$,

(e) $M \models \psi$,

(f) $\left(P^{M},<^{M}\right) \cong\left(\left(2^{\mu}\right)^{+},<\right)$,

(g) $M$ is a $\tau$-model satisfying:

$\left\{\left\{a: a R^{M} b\right\}: b \in M\right\}=[M] \leq \mu$

or just the former family is cofinal in the latter family (both ordered by inclusion).

(B) There are a $\tau$-model $N$ and a sequence $\left\langle b_{n}: n<\omega\right\rangle$ such that:

( $\alpha) N \models \psi$,

( $\beta)\left(P^{N},<^{N}\right)$ is not well ordered,

$(\gamma) b_{n} \in N$ and $N \models(\forall x)\left(x R b_{n} \rightarrow x R b_{n+1}\right)$,

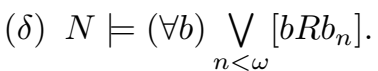

Proof. 1) Follows by 2) of the claim.

2) Without loss of generality, $|M|$ is an ordinal, $P^{M}=\left(2^{\mu}\right)^{+}$and $<^{M}$ is the usual order of the ordinals on $|M|$. By induction on $n$ we choose a sequence $\left\langle\left(M_{n, \gamma}, \bar{\beta}_{n, \gamma}, \bar{b}_{n, \gamma}\right): \gamma<\left(2^{\mu}\right)^{+}\right\rangle$such that

$\boxplus_{1}$ (a) $\quad \bar{\beta}_{n, \gamma}=\left\langle\beta_{n, \gamma, \ell}: \ell<n\right\rangle$ is a decreasing sequence of ordinals from $P^{M}$, which are $>\gamma$ and $\bar{b}_{n, \gamma}=\left\langle b_{n, \gamma, \ell}: \ell<n\right\rangle$ is a sequence of members of $M$.

(b) $\quad M_{n, \gamma} \prec_{\mathbb{L}_{\partial^{+}, \theta^{+}}} M,\left\|M_{n, \gamma}\right\|=\mu$ and $b_{n, \gamma, \ell}, \beta_{n, \gamma, \ell} \in M_{n, \gamma}$ for $\ell<n$ (recall $\mu=2^{\partial}$ ).

(c) For $\gamma_{1}<\gamma_{2}<\left(2^{\mu}\right)^{+}$we have $\left(M_{n, \gamma_{1}}, \bar{b}_{n, \gamma_{1}}, \bar{\beta}_{n, \gamma_{1}}\right) \cong\left(M_{n, \gamma_{2}}, \bar{b}_{n, \gamma_{2}}, \bar{\beta}_{n, \gamma_{2}}\right)$; note that the isomorphism is unique as $<^{M}$ is a well ordering and necessarily the isomorphism maps $b_{n, \gamma_{1}, \ell}$ to $b_{n, \gamma_{2}, \ell}$ and $\beta_{n, \gamma_{1}, \ell}$ to $\beta_{n, \gamma_{2}, \ell}$ for $\ell<n$.

(d) If $n=m+1$, then

(i) $\bar{\beta}_{n, \gamma}\left\lceil m=\bar{\beta}_{m, \zeta}\right.$ and $\bar{b}_{n, \gamma}\left\lceil m=\bar{b}_{m, \zeta}\right.$ when $\zeta=\beta_{n, \gamma, m}$,

(ii) $M_{m, \beta_{n, \gamma, m}} \prec_{\mathbb{L}_{\partial^{+}, \theta^{+}}} M_{n, \gamma}$,

(iii) $a \in M_{m, \beta_{n, \gamma, m}} \Rightarrow M \models a R b_{n, \gamma}$,

(iv) if $b \in M_{m, \beta_{n, \gamma, m}}$, then $\{a: M \models a R b\} \subseteq\left\{a: M \models a R b_{n, \gamma}\right\}$.

For $n=0$ just choose $M_{0, \gamma}=M_{0} \prec_{\mathbb{L}_{\partial^{+}, \theta^{+}}} M,\left\|M_{0}\right\|=\mu$ (O.K. as $\mu^{\partial}=\mu$, in fact an overkill). 
For $n=m+1$, choose $M_{m, \gamma}^{\prime}, b_{m, \gamma}^{\prime}$ such that:

$\boxplus_{2}(i) \quad$ If $a \in M_{m, \gamma}$, then $a R^{M} b_{m, \gamma}^{\prime}$.

(ii) Moreover if $b \in M_{m, \gamma}$, then $\{a \in M: M \models a R b\} \subseteq$

$$
\left\{a \in M: M \models a R b_{m, \gamma}^{\prime}\right\} \text {. }
$$

(iii) $M_{m, \gamma} \cup\left\{b_{m, \gamma}^{\prime}\right\} \cup\{\gamma\} \subseteq M_{m, \gamma}^{\prime}$.

(iv) $\quad M_{m, \gamma}^{\prime} \prec_{\mathbb{L}_{\partial^{+}, \theta^{+}}} M$ and $\left\|M_{m, \gamma}^{\prime}\right\|=\mu$.

The number of $\left(M_{m, \gamma}^{\prime}, M_{m, \gamma}, \bar{\beta}_{m, \gamma+1}, \bar{b}_{n, \gamma+1}, \gamma, b_{m, \gamma}^{\prime}\right) / \cong$ is $\leq 2^{\mu}$, so for some unbounded $Y_{n} \subseteq\left(2^{\mu}\right)^{+}$, the models $\left\langle\left(M_{m, \gamma}^{\prime}, M_{m, \gamma+1}, \bar{\beta}_{m, \gamma+1}, \bar{b}_{n, \gamma+1}, \gamma, b_{m, \gamma}^{\prime}\right): \gamma \in Y_{n}\right\rangle$ are pairwise isomorphic.

For any $\zeta<\left(2^{\mu}\right)^{+}$, let $\gamma_{\zeta}=\operatorname{Min}(Y \backslash(\zeta+1)), M_{n, \zeta}=M_{m, \gamma_{\zeta}}^{\prime}, \bar{b}_{\zeta, n}=\bar{b}_{m, \gamma_{\zeta}}{ }^{\wedge}\left\langle b_{m, \gamma_{\zeta}}^{\prime}\right\rangle$, $\bar{\beta}_{n, \zeta}=\bar{\beta}_{m, \gamma_{\zeta}}{ }^{\wedge}\left\langle\gamma_{\zeta}\right\rangle$. Clearly $\left\langle\left(M_{n, \zeta}, \bar{b}_{n, \zeta}, \bar{\beta}_{n, \zeta}\right): \zeta<\left(2^{\mu}\right)^{+}\right\rangle$is as required.

Having carried the induction, it is easy to find models $N_{n}$ and elements $a_{\ell}, b_{\ell}$ for $\ell<n$ by induction on $n<\omega$ such that:

$\boxplus_{3}(a) \quad\left(N_{n}, a_{0}, \ldots, a_{n-1}, b_{0}, \ldots, b_{n-1}\right)$ is isomorphic to $\left(M_{\gamma, n}, \bar{\beta}_{\gamma, n}, \bar{b}_{\gamma, n}\right)$ for every $\gamma<\left(2^{\mu}\right)^{+}$

(b) $N_{m} \prec_{\mathbb{L}_{\partial^{+}, \theta^{+}}} N_{n}$ if $m<n$.

Now easily by Claim 2.11 the model $N=\bigcup_{n} N_{n}$ and the sequence $\bar{b}=\left\langle b_{n}: n\langle\omega\rangle\right.$ are as required in clause $(\mathrm{B})$ with $\left\langle a_{n}: n<\omega\right\rangle$ witnessing clause $(\beta)$ of clause $(B)$.

Conclusion 3.2. For $\kappa=\beth_{\kappa}$, the logic $\mathbb{L}_{\kappa}^{1}$ satisfies:

$\underline{\text { SUDWO}}_{\kappa}^{1} \quad$ (strong undefinability of well ordering), which means:

If $\psi \in \mathbb{L}_{\kappa}^{1}(\tau),|\tau|<\kappa$ and $<, R$ are two place predicates from $\tau$, then for every large enough $\mu_{1}<\kappa$ for arbitrarily large enough $\mu_{2}<\kappa$ we have:

$\circledast$ If $\lambda>\mu_{2}$ and $\mathfrak{A}$ is a $\tau$-expansion of $\left(\mathscr{H}(\lambda), \in, \mu_{1}, \mu_{2},<\right)$ with $<$ the order on the ordinals and $R^{\mathfrak{A}}$ being $\in$, that is $\in\left\lceil\mathscr{H}(\lambda)\right.$, then we can find $\mathfrak{B}, a_{n}, d_{n}$ (for $n<\omega$ ) such that

(a) $\mathfrak{B} \models \psi \Leftrightarrow \mathfrak{A} \models \psi$,

(b) $\mathfrak{B}=$ " $d_{n+1}<d_{n}<\mu_{2}$ " for $n<\omega$,

(c) $\mathfrak{B} \models$ " $a_{n} \subseteq a_{n+1}$ has cardinality $\leq \mu_{1}$ ",

(d) if $e \in \mathfrak{B}$, then $\mathfrak{B} \models$ "e $e a_{n}$ " for some $n$.

We may add

$(d)^{+} \quad$ if $e \in \mathfrak{B}$ and $\mathfrak{B} \models "|e| \leq \mu_{1}$ ", then $\mathfrak{B} \models$ " $e \subseteq a_{n}$ " for some $n$.

Remark 3.3. There are other variants. At the moment the distinction is not crucial.

Proof. By Claim 3.1.

3.2

Theorem 3.4. First Characterization Theorem

We have $\mathscr{L} \leq \mathbb{L}_{\kappa}^{1}$; i.e. every $\psi \in \mathscr{L}$ is equivalent to some $\psi^{\prime} \in \mathbb{L}_{\kappa}^{1}$ (i.e. for any vocabulary $\tau, \ldots$ ) when:

$\boxplus(a) \quad \kappa=\beth_{\kappa}$ hence is a strong limit uncountable cardinal,

(b) $\mathscr{L}$ is a nice logic,

(c) $\Upsilon$, the occurrence number of $\mathscr{L}$, is $\leq \kappa$, 
(d) $\mathbb{L}_{\kappa}^{-1} \leq \mathscr{L}$, i.e. $\mathbb{L}_{\theta^{+}, \aleph_{0}} \leq \mathscr{L}$ for $\theta<\kappa$ or just

$(d)^{-} \quad$ for every ordinal $\alpha<\kappa$, the model $M_{\alpha}$ expanding $(\alpha,<)$ is characterized up to isomorphism by some sentence from $\mathbb{L}_{\kappa}^{1}\left(\tau\left(M_{\alpha}\right)\right)$, so $\left|\tau\left(M_{\alpha}\right)\right|<\kappa$,

(e) $\mathbb{L}$ satisfies $\mathrm{SUDWO}_{\kappa}^{1}$.

Remark 3.5. 1) We could add the downward LST and restrict somewhat SUDWO .

2) As $\mathbb{L}_{\kappa}^{1}$ satisfies the demands (by Conclusion 2.15) we can rephrase Theorem 3.4 as: $\mathbb{L}$ is $\leq$-maximal such that (a)-(e) hold.

Proof. Assume toward a contradiction that $\psi^{*} \in \mathscr{L}(\tau)$ is a sentence which is not equivalent to any sentence of $\mathbb{L}_{\kappa}^{1}(\tau)$. As the occurrence number of $\mathscr{L}$ is $\leq \kappa$ by clause (c) of the assumption, without loss of generality the vocabulary $\tau$ has cardinality $<\Upsilon \leq \kappa$. Similarly by $\mathscr{L}$ being a logic, using clauses (c),(d) of Definition 1.9. without loss of generality the symbols we add to $\tau$ in $(*)_{3}(a)$ below do not belong to $\tau$. We shall derive another sentence $\psi^{* *}$ in a somewhat larger vocabulary, and let $\left(\mu_{1}, \mu_{2}\right)$ be as in Definition 3.2 for $\psi^{* *}$ and $\mu_{1} \geq \theta_{*}$ where $\theta_{*}=|\tau|+\aleph_{0}$.

Let

$(*)_{0} \quad(a) \quad\left\{P_{i}: i<i(*)\right\}$ list the predicates in $\tau$.

(b) $\left\{H_{j}: j<j(*)\right\}$ list the function symbols in $\tau$.

(c) Let the vocabulary $\tau_{1}$ be

- the set of predicates $\left\{P_{i}^{\prime}: i \leq i(*)\right\}$,

- the set of function symbols $\left\{H_{j}^{\prime}: j<j(*)\right\}$,

- $\operatorname{arity}_{\tau_{1}}\left(P_{i(*)}^{\prime}\right)=2$,

- $\operatorname{arity}_{\tau_{1}}\left(P_{i}^{\prime}\right)=\operatorname{arity}_{\tau}\left(P_{i}\right)+1$ for $i<i(*)$,

- $\operatorname{arity}_{\tau_{1}}\left(H_{j}^{\prime}\right)=\operatorname{arity}_{\tau}\left(H_{j}\right)+1$ for $j<j(*)$.

(d) For a $\tau_{1}$-structure $\mathfrak{B}$ and $c \in \mathfrak{B}$ let $N_{c}^{\mathfrak{B}}$ be the $\tau$-model (if it exists) such that

- it has universe $\left\{d: \mathfrak{B} \models P_{i(*)}^{\prime}(d, d)\right\}$,

- $\left(P_{i}\right)^{N_{c}^{\mathfrak{B}}}=\left\{\bar{a}:\langle c\rangle^{\wedge} \bar{a} \in\left(P_{i}^{\prime}\right)^{\mathfrak{B}}\right\}$ for $i<i(*), j<j(*)$,

- $H_{j}^{N_{c}^{\mathfrak{B}}}(\bar{a})=\left(H_{j}^{\prime}\right)^{\mathfrak{B}}\left(\langle c\rangle^{\wedge} \bar{a}\right)$ for $i<i(*)$.

For each $\alpha<\kappa$, let $\theta(\alpha)=\theta_{\alpha}=|\alpha|+\aleph_{0}$, so the equivalence relation $\mathscr{E}_{\mathrm{qf}(\tau), \theta(\alpha), \alpha}^{1}$ is well defined, and has $<\beth_{\theta(\alpha)}+$ equivalence classes, say $\left\langle N_{\alpha, \varepsilon} / \mathscr{E}_{\mathrm{qf}(\tau), \theta(\alpha), \alpha}: \varepsilon<\right.$ $\left.\varepsilon_{\alpha}<\beth_{\theta(\alpha)}+(|\tau|)\right\rangle$.

By the definition of $\mathbb{L}_{<\theta}^{1}(\tau)$, which is $\leq \mathbb{L}_{\kappa}^{1}(\tau)$, for each such pair $(\alpha, \varepsilon)$ there is a sentence $\vartheta_{\alpha, \varepsilon} \in \mathbb{L}_{\leq \theta(\alpha)}^{1}(\tau)$, which defines $N_{\alpha, \varepsilon} / \mathscr{E}_{\mathrm{qf}(\tau), \theta(\alpha), \alpha}$ and moreover for every $u \subseteq \varepsilon_{\alpha}$ the sentence $\vartheta_{\alpha, u}=\vee\left\{\vartheta_{\alpha, \varepsilon}: \varepsilon \in u\right\}$ belongs to $\mathbb{L}_{\kappa}^{1}(\tau)$, i.e. up to equivalence. Hence by our assumption toward a contradiction for some $\zeta(\alpha)<\varepsilon_{\alpha}$ there are

$$
\begin{aligned}
(*)_{1} & (a) \quad M_{\alpha}, N_{\alpha} \in N_{\alpha, \zeta(\alpha)} / \mathscr{E}_{\mathrm{qf}(\tau), \theta(\alpha), \alpha}^{1}, \\
& (b) \quad N_{\alpha}=\psi^{*} \text { but } M_{\alpha}=\neg \psi^{*} .
\end{aligned}
$$

By the definition of $\mathscr{E}_{\mathrm{qf}(\tau), \theta(\alpha), \theta}$ there is a sequence $\bar{M}_{\alpha}$ such that

$(*)_{2}(a) \quad \bar{M}_{\alpha}=\left\langle M_{\alpha, k}: k \leq k(\alpha)\right\rangle$, so $k(\alpha)<\omega$, 
(b) $M_{\alpha, 0}=M_{\alpha}, M_{\alpha, k(\alpha)}=N_{\alpha}$,

(c) $M_{\alpha, k}, M_{\alpha, k+1}$ are $\mathscr{E}_{\mathrm{qf}(\tau), \theta(\alpha), \alpha^{-}}$-equivalent for $k<k(\alpha)$,

(d) $\quad$ let $M_{\alpha, k} \cong M_{\alpha, k(\alpha)}$ for $k \in(k(\alpha), \omega)$.

Without loss of generality the universes of the models $M_{\alpha, k}(\alpha<\kappa, k<\omega)$ are pairwise disjoint and $\tau \subseteq \mathscr{H}_{<\aleph_{0}}(|\tau|)$. For large enough $\lambda$ we have $\left\langle\left\langle M_{\alpha, k}: k \leq\right.\right.$ $k(\alpha)\rangle: \alpha<\kappa\rangle \in \mathscr{H}(\lambda)$ for every $\alpha<\kappa$ and so $\lambda>\kappa$; let $\mathfrak{A}_{\lambda}=\mathfrak{A}(\lambda)$ be a model such that:

$(*)_{4}(a) \quad$ the vocabulary $\tau_{2}$ of $\mathfrak{A}_{\lambda}$ is $\tau_{1} \cup\left\{<, E, R, R_{1}, F_{1}, F_{2}\right\} \cup\left\{c_{\alpha}: \alpha \leq \theta_{*}^{+}\right\}$, with $c_{\alpha}$ individual constants, $F_{1}, F_{2}$ unary function symbols, $<, E$ binary predicates,

(b) the universe is $\mathscr{H}(\lambda)$,

(c) among the elements $c_{\alpha}^{\mathfrak{A}(\lambda)}\left(\alpha<\theta_{*}\right)$ will be $\kappa, \theta_{*}, \theta_{*}^{+}, \mu_{1}, \mu_{2}, \tau, \tau_{1}$ and every symbol in $\tau$ and $\alpha \leq \theta_{*} \Rightarrow c_{\theta^{*}+\alpha}(\mathfrak{A})=\alpha$; so $c_{0}^{\mathfrak{A}(\lambda)}=\kappa, c_{1}^{\mathfrak{A}(\lambda)}=\theta_{*}$, etc.,

(d) the functions $\omega \alpha+k \mapsto M_{\alpha, k}$ and $\alpha \mapsto k(\alpha)$ are $F_{1}^{\mathfrak{A}(\lambda)}, F_{2}^{\mathfrak{A}(\lambda)}$ respectively,

(e) $<^{\mathfrak{A}(\lambda)}$ is the order on the ordinals,

(f) $\quad E^{\mathfrak{A}(\lambda)}$ is an equivalence relation such that the equivalence classes of $E^{\mathfrak{A}}$ are $\left\{\left|M_{\alpha, k}\right|: \alpha<\kappa\right.$ and $\left.k<\omega\right\}$,

$(g)(\alpha) \quad R^{\mathfrak{A}(\lambda)}=\left\{(a, b): a \in b \in \mathscr{H}(\lambda)\right.$ and $\left.|b| \leq \mu_{1}\right\}$,

(ß) $R_{1}^{\mathfrak{A}(\lambda)}=\in\lceil\mathscr{H}(\lambda)$,

(h) $\left(P_{i(*)}^{\prime}\right)^{\mathfrak{A}(\lambda)}=\left\{(a, \omega \alpha+k): k<\omega, \alpha<\kappa\right.$ and $\left.a \in M_{\alpha, k}\right\}$,

(i) $\left(P_{i}^{\prime}\right)^{\mathfrak{A}(\lambda)}=\left\{\bar{a}^{\wedge}\langle\omega \alpha+k\rangle: \alpha<\kappa, \bar{a} \in P_{i}^{M_{\alpha, k}}\right.$ and $\left.k<\omega\right\}$ for $i<i(*)$,

(j) $H_{j}^{\mathfrak{A}(\lambda)}$ is an $\left(\operatorname{arity}_{\tau}\left(H_{j}\right)+1\right)$-place function satisfying $H_{j}^{\mathfrak{A}}\left(\bar{a}^{\wedge}\langle\omega \alpha+k\rangle\right)=H_{j}^{M_{\alpha, k}}(\bar{a})$ for $j<j(*), \alpha<\kappa$ and $k<\omega$.

Let $\psi^{* *}$ say:

$(*)_{5}(a)$ one first order sentence saying all relevant (set-theoretic) properties,

(b) description of $\tau, \tau_{1}$,

(c) $(\forall \alpha<\kappa)\left(M_{\alpha, 0} \models \psi^{*}\right.$ and $\left.M_{\alpha, k(\alpha)} \models \neg \psi^{*}\right)$.

Why possible? For clauses (a),(b) as $\mathbb{L}_{\kappa}^{-1} \leq \mathscr{L}$, by clause (d) of the assumption or just ensuring $\left\{a: a<c_{1}\right\}$ isomorphic to $\theta_{*}^{+}$if we just assume clause (d) ${ }^{-}$of the assumption and then the rest is said by a first order sentence. For clause (c) as the logic has restriction and weak substitution (see clauses (c),(d) of Definition 1.12) recalling that the logic is closed under the first order operations.

Now apply $\mathrm{SUDWO}_{\kappa}^{1}$ to $\psi^{* *},\left(\mathfrak{A}_{\lambda}, \mu_{1}, \mu_{2}\right)$, and we get $\mathfrak{B}, a_{n}, d_{n}(n<\omega)$ as there.

Now:

$(*)_{6}$ Without loss of generality, $\tau, \tau_{1}$ are interpreted in $\mathfrak{B}$ as $\tau, \tau_{1}$ respectively; similarly the individual constant $c_{\alpha}$ for $\alpha<\theta_{*}^{+}$is interpreted as $c_{\alpha}^{\mathfrak{A}(\lambda)}$. Hence if $\mathfrak{B}=" c$ is an ordinal $<\kappa$ and $k(c)=F_{2}(c)$ ", then for $k<$ $k(c), m \leq k(c)$ we have: 
(a) $M_{c, m}^{\mathfrak{B}}$ defined naturally as $N_{F_{1}^{\mathfrak{B}}(c, m)}^{\mathfrak{B}}$ is a $\tau$-model (i.e. no non-standard predicates!),

(b) $M_{c, 0}^{\mathfrak{B}} \models \psi$ and $M_{c, k(c)}^{\mathfrak{B}} \models " \neg \psi "$,

(c) $\mathfrak{B} \models$ "in the game $\partial_{\mathrm{qf}(\tau), \theta_{*}, c}\left[M_{c, k}^{\mathfrak{B}}, M_{c, k+1}^{\mathfrak{B}}\right]$ the ISO player wins".

$(*)_{7}$ Let $k(*)=F_{2}^{\mathfrak{B}}\left(d_{0}\right)$.

$(*)_{8}$ We fix a winning strategy $\mathbf{s t}_{k}$ for ISO in $\mathfrak{B}$ 's sense in the game $\partial_{\mathrm{qf}(\tau), \theta_{*}, d}\left[N_{d_{0}, k}^{\mathfrak{B}}, N_{d_{0}, k+1}^{\mathfrak{B}}\right]$.

Now for each $k<k(*)$ by induction on $n<\omega$ we choose $\mathbf{s}^{n, k} \in \mathfrak{B}$ such that:

$(*)_{9}(a) \quad \mathfrak{B} \models \cdot \overline{\mathbf{s}}^{n, k}=\left\langle\mathbf{s}_{0, k}, \ldots, \mathbf{s}_{n, k}\right\rangle$ is an initial segment of a play of the game $\partial_{\mathrm{qf}(\tau), \theta_{*}, d_{0}}\left[M_{d_{0}, k}^{\mathfrak{B}}, M_{d_{0}, k+1}^{\mathfrak{B}}\right] "$.

(b) $\mathfrak{B}=$ "in this initial segment $\mathbf{s}^{n, k}$ the ISO player uses his winning strategy $\mathbf{s t}_{k} "$.

(c) The AIS player chooses:

$(\alpha) \quad$ if $n$ is even, then $\iota_{n}=1$ and $A_{n}^{\prime}=\left\{e \in \mathfrak{B}: e \in M_{d_{0}, k}^{\mathfrak{B}}\right.$,

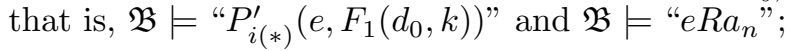

( $\beta) \quad$ if $n$ is odd, then $\iota_{n}=2$ and $A_{n}^{\prime}=\left\{e \in \mathfrak{B}: e \in M_{d_{0}, k+1}^{\mathfrak{B}}\right.$ and $\mathfrak{B} \models$ "eRan $\}$ ";

$(\gamma)$ the "ordinal" $\beta_{\mathbf{s}_{n, k}}=d_{n+1}$.

This can be done and $g_{k}=\left\{\left(a_{1}, a_{2}\right)\right.$ : for some $n$ we have $\left.\mathfrak{B} \models " g_{n}^{\mathbf{s}_{n, k}}\left(a_{1}\right)=a_{2} "\right\}$; it is an isomorphism from $M_{d_{0}, k}^{\mathfrak{B}}$ onto $M_{d_{0}, k+1}^{\mathfrak{B}}$. As this holds for every $k<k(*)$ we get that $M_{d_{0}, 0}^{\mathfrak{B}}, M_{d_{0}, k(*)}^{\mathfrak{B}}$ are isomorphic, so by $(*)_{6}(b)$ we get a contradiction. $\square$

Definition 3.6. Let $\Theta_{\theta, R}$ be the sentence (for $R$ a binary predicate) such that $M=\left(|M|, R^{M}\right) \models \Theta_{\theta, R}$ iff $\mathscr{P}=\left\{\left\{a: a R^{M} b\right\}: b \in M\right\}$ is a $\left(\theta, \aleph_{0}\right)$-cover of $\operatorname{Dom}\left(P^{M}\right)$ which means: it is a family of subsets of $\operatorname{Dom}(R)=\left\{a: a R^{M} b\right.$ for some $b \in M\}$ each of cardinalilty $\leq \theta$ such that any $u \in\left[\operatorname{Dom}\left(R^{M}\right)\right] \leq \theta$ is included in the union of countably many such sets.

Claim 3.7. 1) $\Theta_{\theta, R}$ can be expressed by a sentence in $\mathbb{L}_{\leq \theta^{+}}^{1}(\{R\})$.

2) In fact, if $M_{1}, M_{2}$ are $\mathscr{E}_{\mathrm{qf}(\{R\}), \theta^{+}, \omega+\omega+1^{-e q u i v a l e n t}}\{R\}$-models, then $M_{1} \models$ $\Theta_{\theta, R} \Leftrightarrow M_{2} \models \Theta_{\theta, R}$.

3) If $M_{1} \mathscr{E}_{\mathrm{qf}(\tau), \theta, \alpha} M_{2}$ and $\alpha \geq \omega+\omega+1$ and $\operatorname{cf}(\theta) \geq \aleph_{0}$, then $\left\|M_{1}\right\|=\left\|M_{1}\right\|$ or $\left\|M_{1}\right\|,\left\|M_{2}\right\|>\theta$.

4) If $M_{1} \mathscr{E}_{\mathrm{qf}(\tau), \theta, \alpha}^{1} M_{2}$ and $\left\|M_{1}\right\| \leq \theta$ and $\alpha \geq \omega+\omega+1$, then $M_{1} \cong M_{2}$.

Proof. 1) By 2) of the claim.

2) Toward a contradiction, assume this fails; without loss of generality, $M_{1} \mathscr{E}_{\mathrm{qf}(\{R\}), \theta^{+}, \omega+\omega+1} M_{2}$. By symmetry, without loss of generality, assume $M_{2} \models$ $\Theta_{\theta, R}$ but $M_{1} \models \neg \Theta_{\theta, R}$. We simulate a play of the game $\partial_{\mathrm{qf}(\{R\}), \theta^{+}, \omega+\omega+1}\left(M_{1}, M_{2}\right)$ in which the ISO player uses a (fixed) winning strategy st.

Case 1: There is $B_{*} \in\left[M_{1}\right] \leq \theta$ not included in a countable union of sets from $\left\{\left\{a: a R^{M_{1}} b\right\}: b \in M_{1}\right\}$. Here $\partial_{\mathrm{qf}(\{R\}), \theta, \omega+\omega+1}\left[M_{1}, M_{2}\right]$ suffices; we now simulate a play in which the ISO player uses st. 
In the first move we let the AIS player choose $\beta_{\mathbf{s}_{1}}=\omega+\omega$ and choose $\iota_{\mathbf{s}_{1}}=$ $1, A_{\mathbf{s}_{1}}^{1}=B_{*} \in\left[M_{1}\right]^{\leq \theta}$, see above. Let the ISO player (using st) complete the choice of the state $\mathbf{s}_{1}$ (which is a winning state itself, of course).

Let $n(*)$ be minimal such that:

$(*) B:=\left\{a \in A_{\mathbf{s}_{1}}^{1}: h_{\mathbf{s}_{1}}^{1}(a)<n(*)-7\right\}$ is not included in a countable union of sets from $\left\{\left\{a: a R^{M_{1}} b\right\}: b \in B\right\}$.

By the case assumption, $n(*)$ is well defined. In the following $n(*)-2$ moves, the AIS player takes care that $\beta_{\mathbf{s}_{2}}=\omega+n(*), \ldots, \beta_{\mathbf{s}_{n(*)}}=\omega+1$ (and $\iota_{\mathbf{s}_{2}}=\cdots=\iota_{\mathbf{s}_{n(*)}}=1$ ).

So $g_{\mathbf{s}_{n(*)}}$ is a function whose domain includes $\left\{a \in A_{\mathbf{s}_{1}}^{1}: h_{\mathbf{s}_{1}}^{1}(a)<n(*)\right\}$, hence includes $B$. Now as $M_{2} \models \Theta_{\theta, R}$ we can find $c_{n} \in M_{2}$ for $n<\omega$ such that $(\forall b \in$ B) $\bigvee_{n} g_{\mathbf{s}_{n(*)}}(b) R^{M_{2}} c_{n}$ and AIS player takes care that $\beta_{\mathbf{s}_{n(*)+1}}=\omega$ and $\left\{c_{n}: n<\right.$ $\omega\} \subseteq A_{\mathbf{s}_{n(*)+1}}^{2}$.

The rest should be clear.

Case 2: There is $b_{*} \in M_{1}$ such that $B_{*}=\left\{a \in M_{1}: a R^{M_{1}} b_{*}\right\}$ has cardinality $>\theta$.

Here $\partial_{\mathrm{qf}(\{R\}), \theta^{+}, \omega+1}$ suffices, and let $B \subseteq B_{*}$ be of cardinality $\theta^{+}$. We simulate a play in which the AIS player takes care that $\beta_{\mathbf{s}_{1}}=\omega, \iota_{\mathbf{s}_{1}}=1, A_{\mathbf{s}_{1}}^{1}=\left\{b_{*}\right\} \cup B$. $\theta^{+}$.

We let $n_{*}$ be minimal such that $h_{\mathbf{s}_{1}}^{1}\left(b_{*}\right)<n_{*}-7$ and $\left|\left\{b \in B: h_{\mathbf{s}_{1}}^{1}(b)<n_{*}-7\right\}\right| \geq$

The rest should be clear.

3), 4) Should be clear.

Recall

Definition 3.8. 1) A logic $\mathscr{L}$ is $\Delta$-closed when: for vocabularies $\tau_{1} \subseteq \tau_{2}$ with $\tau_{2} \backslash \tau_{1}$ finite, and sentences $\psi, \vartheta \in \mathscr{L}\left(\tau_{2}\right)$ if $\overline{K_{0}=}\left\{M\left\lceil\tau_{1}: M \models \psi\right\}, K_{1}=\left\{M \uparrow \tau_{1}\right.\right.$ : $M \models \vartheta\}$ are complementary classes of $\tau_{1}$-models, then some $\varphi \in \mathscr{L}\left(\tau_{1}\right)$ define $K_{1}$.

2) A logic $\mathscr{L}$ is strongly $\Delta$-closed when: for relational vocabularies $\tau_{1} \subseteq \tau_{2}$ with $\tau_{2} \backslash \tau_{1}$ finite, unary predicate $P \in \tau_{2} \backslash \tau_{1}$ and sentences $\psi, \vartheta \in \mathscr{L}\left(\tau_{2}\right)$, if $K_{0}:=$ $\left\{\left(M\left\lceil\tau_{1}\right)\left\lceil P^{M}: M \models \psi\right.\right.\right.$ and $\left.P^{M} \neq \emptyset\right\}$ and $K_{1}:=\left\{\left(M\left\lceil\tau_{1}\right)\left\lceil P^{M}: M \models \vartheta\right.\right.\right.$ and $\left.P^{M} \neq \emptyset\right\}$ are complementary classes of $\tau_{1}$-models, then some $\varphi \in \mathscr{L}\left(\tau_{1}\right)$ define $K_{1}$.

3) A logic $\mathscr{L}$ has dullness-elimination when: if $\tau_{1}, \tau_{2}$ are relational vocabularies, i.e. with predicates only, $\tau_{2}=\tau_{1} \cup\{P\}, P$ a unary predicate, $\psi_{2} \in \mathscr{L}\left(\tau_{2}\right)$ and for every $M=\psi_{2}$ we have $Q \in \tau_{1} \Rightarrow Q^{M}=Q^{M}\left\lceil P^{M}\right.$ and $P^{M} \neq \emptyset$, then for some $\psi_{1} \in \mathscr{L}\left(\tau_{1}\right)$ we have $\left\{M: M\right.$ a $\tau_{1}$-model of $\left.\psi_{1}\right\}=\left\{\left(M\left\lceil\tau_{1}\right) \uparrow P^{M}: M\right.\right.$ a $\tau_{2}$-model of $\left.\psi_{2}\right\}$.

4) We say that the logic $\mathscr{L}$ is $\Delta$-closed in $\mathbf{C}$ for $\mathbf{C}$ a class of cardinals when: if in part (1) we assume just that $K_{0} \cap K_{\mathbf{C}}^{\tau}, K_{0} \cap K_{\mathbf{C}}^{\tau}$ are complementary in $K_{\mathbf{C}}^{\tau}=\{M: M$ a $\tau$-model of cardinality from $\mathbf{C}\}$, then for some $\psi \in \mathscr{L}\left(\tau_{1}\right)$ for every $\tau_{1}$-model $M$ of cardinality $\in \mathbf{C}$ we have $M \models \psi \Leftrightarrow M \in K_{0}$.

Claim 3.9. 1) The logic $\mathbb{L}_{<\theta}^{1}$ has dullness-elimination.

2) If $\tau$ is empty, $M_{1}, \bar{M}_{2}$ are $\tau$-models of cardinality $>\theta$, then $M_{1}, M_{2}$ are $\mathscr{E}_{\mathrm{qf}(\tau), \theta, \alpha}$-equivalent for $\alpha<\theta^{+}$.

3) Also $\left\|M_{1}\right\|,\left\|M_{2}\right\| \geq \theta$ and $\operatorname{cf}(\theta)=\aleph_{0}$ is O.K. in part 2) of Claim 3.9

Proof. 1) Easy (or use the sum theorem (see Theorem 3.16) and part 2) of Claim 3.9 .

2), 3) Easy. 
Theorem 3.10. Second Characterization Theorem

Let $\kappa=\beth_{\kappa}$. Then $\mathbb{L}_{\kappa}^{1}$ is the minimal logic $\mathscr{L}$ (up to equivalence) such that:

$\oplus$ (a) $\mathscr{L}$ is a nice logic,

(b) $\mathbb{L}_{\kappa}^{-1} \leq \mathscr{L}$, i.e. $\mathbb{L}_{\theta^{+}, \aleph_{0}} \leq \mathscr{L}$ for $\theta<\kappa$,

(c) $\Theta_{\theta, R}$ is equivalent to some sentence in $\mathscr{L}$ for $\theta<\kappa$,

(d) $\mathscr{L}$ is $\Delta$-closed,

(e) $\mathscr{L}$ has dullness-elimination.

Remark 3.11. Putting together Theorems 3.4, 3.10 we get full characterization.

Proof. First $\mathbb{L}_{\kappa}^{1}$ satisfies clause (a) by Conclusion 2.15(2), clause (b) by Conclusion 2.15(3), clause (c) by Claim 3.7(1) and clause (d) by Theorem 3.13 and Observation 3.12(1) below, and lastly $\mathbb{L}_{\kappa}^{1}$ has dullness-elimination by Claim 3.9 (1), so together $\mathbb{L}_{\kappa}^{1}$ satisfies $\oplus$ of Theorem 3.10 . Second, we shall assume that $\mathscr{L}$ satisfies $\oplus$ and $\psi \in \mathbb{L}_{\kappa}^{1}(\tau)$ and we shall find $\psi^{\prime} \in \mathscr{L}(\tau)$ equivalent to it (it will be $\varphi_{1}$ below). By "L $\mathscr{L}$ satisfies weak substitution" without loss of generality $\tau$ has predicates only.

We deal with and define naturally $\psi^{[P]} \in \mathbb{L}_{\kappa}^{1}(\tau), P$ a new unary predicate and let $\tau^{\prime}=\tau \cup\{P\}$, so $\psi^{[P]}$ says that $\left(M\lceil\tau)\left\lceil P^{M}\right.\right.$ satisfies $\psi$.

Let $\theta, \alpha<\kappa$ and let $\psi \in \mathbb{L}_{\kappa}^{1}(\tau)$ be defined using $(\operatorname{qf}(\tau), \theta, \alpha)$, so $|\tau|<\kappa$. We let $\partial=\beth_{\alpha+1}(\theta+|\tau|)$ and $\mu=2^{\partial}$. Let $\tau^{+}=\tau \cup\left\{R_{1}, R\right\}$, $\operatorname{arity}\left(R_{1}\right)=3$, $\operatorname{arity}(R)=2$ and $R, R_{1} \notin \tau$. For $i=0,1$ let $K_{\ell}$ be the class of $\tau^{+}$-models $M$ such that:

$\boxplus_{\ell}(a) \quad\left(|M|, R^{M}\right) \models \Theta_{\mu, R}$ and $P^{M} \neq \emptyset$.

(b) $\left\{B_{b}: b \in M\right\}$ is $\subseteq$-directed where $B_{b}=\left\{a \in M: a R^{M} b\right\}$.

(c) For each $b \in M$ the set $\left\{\left(a_{1}, a_{2}\right):\left(a_{1}, a_{2}, b\right) \in R_{1}^{M}\right\}$ is a well ordering of $B_{b}$ of order type $\leq \mu$.

(d) If $b_{1}, b_{2} \in M$ and $B_{b_{1}} \subseteq B_{b_{2}} \subseteq P^{M}$, then $(M \uparrow \tau)\left\lceil B_{b_{1}} \prec \mathbb{L}_{\partial^{+}, \partial^{+}}(M \uparrow \tau)\left\lceil B_{b_{2}}\right.\right.$; in particular $B_{b_{1}}$ is closed under $F^{M}$ for any function symbol $F \in \tau$.

(e) If $b \in M$, then for some $c$ we have $B_{c}=B_{b} \cap P^{M} \subseteq B_{c} \subseteq P^{M}$.

(f) If $b \in M$ and $B_{b} \subseteq P$, then $\left(M\lceil\tau) \mid B_{b} \models \psi^{[P]}\right.$ iff $\ell=1$.

(g) If $Q \in \tau$, then $Q^{M}=Q^{M}\left\lceil P^{M}\right.$.

Now:

$(*)_{1}$ Clauses (b),(c),(d),(e),(g) of $\boxplus_{\ell}$ can be described by some $\varphi \in \mathbb{L}_{\left(2^{\mu}\right)+, \aleph_{0}}\left(\tau_{1}\right)$.

[Why? Note clause (c), so obvious.]

$(*)_{2}$ Clause (f) can be expressed by a sentence from $\mathscr{L}\left(\tau^{+}\right)$.

[Why? Let $\left\langle N_{\alpha}: \alpha<2^{\mu}\right\rangle$ list the $\tau$-models with universe $\subseteq \mu$. For each $b \in M$ such that $B_{b} \subseteq P^{M}$ let $g_{b}$ be the unique one-to-one function from $B_{b}$ onto some ordinal $\gamma_{b} \leq \mu$ such that

- if $a_{1}, a_{2} \in B_{b}$, then $\left(a_{1}, a_{2}, b\right) \in R_{1}^{M} \Leftrightarrow g_{b}(a)<g_{b}\left(a_{2}\right)$. 
Hence for such a $b$ there is a unique $\alpha_{b}$ such that $g_{b}$ is an isomorphism from $\left(M\lceil\tau)\left\lceil B_{b}\right.\right.$ onto $N_{\alpha}$. For each $\alpha$ there is a formula $\psi_{\alpha}(x) \in \mathbb{L}_{\mu^{+}, \aleph_{0}}\left(\tau_{M}\right)$ such that for every $b \in M$ we have $M \models \psi_{\alpha}[b]$ iff $b$ is as above and $\alpha_{b}=\alpha$.

So for every $u \subseteq 2^{\mu}, \psi_{u}^{\prime}(x)=\bigvee_{\alpha \in u} \psi_{\alpha}(x)$ belongs to $\mathbb{L}_{\left(2^{\mu}\right)^{+}, \aleph_{0}}\left(\tau_{M}\right)$, hence is equivalent to some $\psi_{u}^{\prime \prime}(x) \in \mathscr{L}\left(\tau_{M}\right)$. Now $(*)_{2}$ should be clear.]

$(*)_{3} K_{\ell}$ is definable by some $\varphi_{\ell} \in \mathscr{L}\left(\tau^{+}\right)$for $\ell=1,2$.

[Why? By clause (b) of the assumption, some $\varphi^{\prime} \in \mathscr{L}\left(\tau^{+}\right)$is equivalent to $\varphi$, where $\varphi$ is from $(*)_{1}$. By clause (c) of the assumption some $\varphi^{\prime \prime} \in \mathscr{L}\left(\tau^{+}\right)$is equivalent to $\Theta_{\theta, R}$. But by clause (a) of the assumption $\mathscr{L}$ is a nice logic; hence (see clauses $(b)_{2},(b)_{3},(c)$ of Definition 1.12) there is a sentence $\varphi_{\ell}^{\prime} \in \mathscr{L}\left(\tau^{+}\right)$equivalent to $\varphi_{\ell}$ from $(*)_{2}$. By clause $(b)_{2}$ of Definition 1.12, there is $\varphi_{\ell} \in \mathscr{L}\left(\tau^{+}\right)$equivalent to $\varphi^{\prime} \wedge \varphi^{\prime \prime} \wedge \varphi_{\ell}^{\prime}$, so we are done.]

$(*)_{4}$ For $\ell=1,2$ the classes $\left\{\left(M_{1}\lceil\tau)\left\lceil P^{M_{1}}: M_{1} \in K_{1}\right\},\left\{\left(M_{2}\lceil\tau) P_{i}^{M}, M_{2} \in\right.\right.\right.\right.$ $\left.K_{2}\right\}$ are equal to $\{M: M$ a $\tau$-model of $\psi\},\{M: M$ a $\tau$-model of $\neg \psi\}$, respectively.

By $\mathscr{L}$ being $\Delta$-closed and having dullness-elimination (see clauses (d), (e) of the theorem's assumption) we are done.

We can note and recall

Observation 3.12. 1) If the logic $\mathscr{L}$ satisfies interpolation, then it is $\Delta$-closed.

2) $\mathscr{L}$ is $\Delta$-closed and has dullness-elimination iff $\mathscr{L}$ is strongly $\Delta$-closed.

3) If $\mathscr{L}$ satisfies interpolation with finitely many sorts (so the interpolant mentions only the common sorts), then $\mathscr{L}$ is strongly $\Delta$-closed.

4) The logic $\mathscr{L}$ satisfies interpolation and dullness-elimination iff $\mathscr{L}$ satisfies interpolation for finitely many sort models.

5) In Theorem 3.10 we can replace clauses (d),(e) by

$(d)^{\prime}$ for every $\mu<\kappa, \mathscr{L}$ is $\Delta$-closed in $\left\{\lambda: \lambda=\lambda^{\mu}\right\}$; see Definition 3.8

Proof. Should be clear.

Theorem 3.13. 1) For $\kappa=\beth_{\kappa}$ the logic $\mathbb{L}_{\kappa}^{1}$ satisfies interpolation.

2) Also if $\psi \in \mathbb{L}_{\leq \theta}^{1}\left(\tau_{1}\right)$ where $\theta<\kappa$, the vocabularies $\tau_{0} \subseteq \tau_{1}$ have cardinality $\leq \theta$ and $\partial=\beth_{\theta^{+}}, \mu=2^{\partial}$, then we can find a sequence $\left\langle\psi_{\alpha}: \alpha<\left(2^{\partial}\right)^{+}\right\rangle$of members of $\mathbb{L}_{\leq 2^{\partial}}^{1}\left(\tau_{0}\right)$ such that:

(a) $\vdash \psi \rightarrow \psi_{\alpha}$ for $\alpha<\left(2^{\partial}\right)^{+}$,

(b) if $\tau_{0}=\tau_{1} \cap \tau_{2}$ and $\vartheta \in \mathbb{L}_{\leq \theta}^{1}\left(\tau_{2}\right)$ a sentence such that $\vdash \psi \rightarrow \vartheta$, then $\vdash \psi_{\alpha} \rightarrow \vartheta$ for some $\alpha<\left(2^{\partial}\right)^{+}$.

Proof. 1) This means that we should prove the existence of $\psi \in \mathbb{L}_{\kappa}^{1}\left(\tau_{0}\right)$ such that $\vdash \psi_{1} \rightarrow \psi$ and $\vdash \psi \rightarrow \psi_{2}$ when we assume:

$$
\begin{aligned}
\boxplus_{1} & (a) \quad \tau_{1} \cap \tau_{2}=\tau_{0}, \\
& (b) \quad \psi_{\ell} \in \mathbb{L}_{\kappa}^{1}\left(\tau_{\ell}\right) \text { for } \ell=1,2, \\
& \text { (c) } \vdash \psi_{1} \rightarrow \psi_{2} .
\end{aligned}
$$


Toward a contradiction we assume this fails. Without loss of generality, $\tau_{\ell}(\ell<3)$ are vocabularies of cardinality $<\kappa$.

Let $\theta_{*}$ be such that $\theta_{*}<\kappa, \theta_{*} \geq\left|\tau_{1}\right|+\left|\tau_{2}\right|$ and $\psi_{\ell} \in \mathbb{L}_{\leq \theta_{*}}^{1}$ for $\ell=1,2$. For each $\theta \in\left[\theta_{*}, \kappa\right)$ as $\mathbb{L}_{<\theta}^{1}\left(\tau_{0}\right)$ is closed under conjunction (and conjunctions) of $\leq \theta$ formulas and negations, clearly

$\boxplus_{2}$ for every $\alpha<\kappa$ we can choose $M_{\alpha}^{1}, M_{\alpha}^{2}$ such that

(a) $M_{\alpha}^{1}$ is a $\tau_{1}$-model,

(b) $M_{\alpha}^{2}$ is a $\tau_{2}$-model,

(c) $M_{\alpha}^{1}\left\lceil\tau_{0}, M_{\theta}^{2}\left\lceil\tau_{0}\right.\right.$ are $\mathscr{E}_{\mathrm{qf}\left(\tau_{0}\right),|\alpha|, \alpha}^{1}$-equivalent,

(d) $M_{\alpha}^{1} \models \psi_{1}$,

(e) $M_{\alpha}^{2} \models \neg \psi_{2}$,

and continue naturally as in the proof of Theorem 3.4

2) Similarly.

Theorem 3.14. If $\kappa=\beth_{\kappa}$, then $\mathbb{L}_{\kappa}^{1}$ satisfies the addition and product theorems, that is (all the models are $\tau$-models for a fixed vocabulary $\tau$; for the sum case we assume $\tau$ has no function symbols (in particular no individual constants or only unary functions) and the relevant models have disjoint universes):

(a) sum: $\operatorname{Th}_{\mathbb{L}_{\kappa}^{1}}\left(M_{1}+M_{2}\right)=\operatorname{Th}_{\mathbb{L}_{\kappa}^{1}}\left(M_{1}\right)+\operatorname{Th}_{\mathbb{L}_{\kappa}^{1}}\left(M_{2}\right)$, that is,

$(a)^{\prime}$ if $\operatorname{Th}_{\mathbb{L}_{\kappa}^{1}}\left(M_{\ell}\right)=\operatorname{Th}_{\mathbb{L}_{\kappa}^{1}}\left(N_{\ell}\right)$ for $\ell=1,2$, then $\operatorname{Th}_{\mathbb{L}_{\kappa}^{1}}\left(M_{1}+M_{2}\right)=\operatorname{Th}_{\mathbb{L}_{\kappa}^{1}}\left(N_{1}+\right.$ $N_{2}$ ),

(b) product: $\operatorname{Th}_{\mathbb{L}_{\kappa}^{1}}\left(M_{1} \times M_{2}\right)=\operatorname{Th}_{\mathbb{L}_{\kappa}^{1}}\left(M_{1}\right) \times \operatorname{Th}_{\mathbb{L}_{\kappa}^{1}}\left(M_{2}\right)$, that is,

$(b)^{\prime}$ if $\operatorname{Th}_{\mathbb{L}_{\kappa}^{1}}\left(M_{\ell}\right)=\operatorname{Th}_{\mathbb{L}_{\kappa}^{1}}\left(N_{\ell}\right)$ for $\ell=1,2$, then $\operatorname{Th}_{\mathbb{L}_{\kappa}^{1}}\left(M_{1} \times M_{2}\right)=\operatorname{Th}_{\mathbb{L}_{\kappa}^{1}}\left(N_{1} \times\right.$ $N_{2}$ ),

(c) moreover, we can replace $\operatorname{Th}_{\mathbb{L}_{\kappa}^{1}}(N)$ by $N / \mathscr{E}_{\mathrm{qf}(\tau), \theta, \alpha}^{1}$ when $\theta<\kappa, \alpha<\theta^{+}$.

Proof. It suffices to prove clause (c). We prove it for products. For the sums this is easier.

Clause (c) for products:

Clearly it suffices to prove:

$\boxplus$ Assume $M_{\iota}=M_{1}^{\iota} \times M_{2}^{\iota}$ for $\iota=1,2$ are $\tau$-models and $M_{\ell}^{1} \mathscr{E}_{\mathrm{qf}(\tau), \theta, \alpha}^{1} M_{\ell}^{2}$ for $\ell=1,2$. Then $M_{1} \mathscr{E}_{\mathrm{qf}(\tau), \theta, \alpha}^{1} M_{2}$.

So let $\left\langle M_{\ell, k}: k \leq k(\ell, \iota)\right\rangle$ be such that $M_{1,0}=M_{\ell}^{1}, M_{2, k}=M_{\ell}^{2}$ and $M_{\ell, k} \mathscr{E}_{\mathrm{qf}(\tau), \theta, \alpha}^{0} M_{\ell, k+1}$ for $k<k(\ell)$. Let $k(*)=\max \{k(1), k(2)\}$ and let $M_{\ell, k}=$ $M_{\ell, k(\ell)}$ if $k(\ell)<k \leq k(*)$. As $\mathscr{E}_{\mathrm{qf}(\tau), \theta, \alpha}^{0}$ is reflexive, we still have $M_{\ell, k} \mathscr{E}_{\mathrm{qf}(\tau), \alpha}^{0} M_{\ell, k+1}$ for $\ell=1,2$ and $k<k(*)$.

Letting $M_{k}=M_{1, k} \times M_{2, k}$ for $k \leq k(*)$ we have to prove $M_{0} \mathscr{E}_{\mathrm{qf}(\tau), \theta, \alpha}^{1} M_{k(*)} ;$ by the definition of $\mathscr{E}_{\mathrm{qf}(\tau), \theta, \alpha}^{1}$ it suffices to prove for each $k<k(*)$ that

$\oplus_{k} \quad M_{k} \mathscr{E}_{\mathrm{qf}(\tau), \theta, \alpha}^{0} M_{k+1}$. 
So we have to find a winning strategy for the ISO player for the game $\partial_{\mathrm{qf}(\tau), \theta, \alpha}\left[M_{k}, M_{k+1}\right]$.

The ISO player restricts itself in the $n$-th move to $(\theta, n)$-states $\mathbf{s}_{n}$ for a play of $\partial_{\mathrm{qf}(\tau), \theta, \alpha}\left[M_{k}, M_{k+1}\right]$ satisfying, for some pair $\left(\mathbf{s}_{n, 1}, \mathbf{s}_{n, 2}\right)$, that for $\ell=1,2$,

(a) $\mathbf{s}_{n, \ell}$ is a winning $n$-state for the game $\partial_{\mathrm{qf}(\tau), \theta, \alpha}\left[M_{\ell, k}, M_{\ell, k+1}\right]$,

(b) $A_{\mathbf{s}_{n}}^{\ell}=A_{\mathbf{s}_{n, 1}}^{\ell} \times A_{\mathbf{s}_{n, 2}}^{\ell}$,

(c) $h_{\mathbf{s}_{n}}((b, c))=\max \left\{h_{\mathbf{s}_{n, 1}}^{1}(b), h_{\mathbf{s}_{n, \ell}}^{2}(c)\right\}$.

The rest should be clear.

Remark 3.15. 1) Theorem 3.14(a) applies to the monadic version, too.

2) Why $\kappa=\beth_{\kappa}$ ? As for any $\theta$ and $\alpha<\theta^{+}$there is a sentence $\psi \in \mathbb{L}_{\theta^{+}}$and model $M_{\psi}$ of $\psi$ of cardinality $\beth_{\alpha}$ such that any other model $N$ of $\psi$ can be embedded into $M_{\psi}$.

3) For infinite addition, i.e. $\sum_{s \in I} M_{s}^{\ell}$, we have a problem: passing to $\mathscr{E}_{\mathrm{qf}(\tau), \theta, \alpha}^{0}$ we do not know how to uniformize $k(s)$, of course: if $k(s)$ is constant or bounded there, then a parallel claim holds; still see Theorem 3.16 below.

4) Even overcoming this obstacle, for infinite products we have a problem during a play of $\partial_{\mathrm{qf}(\tau), \theta, \alpha}[M, N]$. The point is about translating the $h$ 's from here to there and back. There may well be a difference between products (or reduced products) and generalized products. Anyhow unlike part (2) this cannot be remedied using $\mathscr{E}_{\mathrm{qf}(\tau), \theta, \alpha}^{0}$ and we know that; see Theorem 4.10.

Theorem 3.16. 1) Assume, for simplicity, that $\tau$ is a relational vocabulary and $\left\langle M_{s}^{\ell}: s \in I\right\rangle$ is a sequence of $\tau$-models with pairwise disjoint universes, for $\ell=1,2$ and $M_{1}=\sum\left\{M_{s}^{1}: s \in S\right\}, M_{2}=\sum\left\{M_{s}^{2}: s \in S\right\}$. If $\kappa=\beth_{\kappa}$ and $M_{s}^{1}, M_{s}^{2}$ are $\mathbb{L}_{\kappa}^{1}$-equivalent for $s \in S$, then $M_{1}, M_{2}$ are $\mathbb{L}_{\kappa}^{1}$-equivalent.

2) Moreover for every $\theta, \alpha<\kappa$ there are $\partial, \beta<\kappa$ such that for any set $S$ and sequence $\left\langle M_{s}^{\ell}: s \in S\right\rangle$ for $\ell \in\{1,2\}$ of pairwise disjoint $\tau$-models we have: if $M_{s}^{1}, M_{s}^{2}$ are $\mathscr{E}_{\mathrm{qf}(\tau), \partial, \beta}$-equivalent for $s \in S$, then the models $M_{1}=\sum\left\{M_{s}^{1}: s \in\right.$ $S\}, M_{2}=\sum\left\{M_{s}^{2}: s \in S\right\}$ are $\mathscr{E}_{q f(\tau), \theta, \alpha \text {-equivalent. }}$

3) In part 2) above if $M_{s}^{b} \mathscr{E}_{\mathrm{qf}(\tau), \theta, \alpha}^{0} M_{j}^{2}$ for every $s \in S$, then $M_{1} \mathscr{E}_{\mathrm{qf}(\tau), \theta, \alpha}^{0} M_{2}$.

Proof. 1), 2) By the proof of Theorem 3.4.

3) As in the proof of Theorem 3.14

Remark 3.17. This proof indicates that there are better versions of the game for which we can choose $(\partial, \beta)=(\theta, \alpha)$. However, it is not clear whether a more complicated definition is worth the gain.

\section{4. $\mathbb{L}_{\kappa}^{1}$ IS STRONG}

Our first aim is:

Question 4.1. How strong is $\mathbb{L}_{\kappa}^{1}$ ? Is it more like $\mathbb{L}_{\kappa}^{-1}$ or $\mathbb{L}_{\kappa}^{0}$ ? E.g. upward LST? Downward LST to which cardinals?

So far we have given an indication to its being similar to $\mathbb{L}_{\kappa}^{-1}$; however we shall show below that for the LST, $\mathbb{L}_{\kappa}^{1}$ is closer to $\mathbb{L}_{\kappa}^{0}$ (see Claim4.8) and fails the theorem on the theory of an infinite product (which both $\mathbb{L}_{\kappa}^{0}, \mathbb{L}_{\kappa}^{-1}$ satisfy). But restricting ourselves to cardinals $\lambda=\lambda^{\aleph_{0}} \geq \kappa$, the situation for the downward LST is similar to the one for $\mathbb{L}_{\kappa}^{0}$ : see below. 
Definition 4.2. For a relational vocabulary $\tau$ and cardinal $\theta$ of cofinality $>\aleph_{0}$ we say that $\mathbf{K}$ is a $(\tau, \theta, \omega)$-class of structures when:

(a) $\mathbf{K}$ is a class of $\tau$-models each of cardinality $\theta$,

(b) $\mathbf{K}$ is closed under submodels of cardinality $\theta$,

(c) $\mathbf{K}$ is closed under isomorphism,

(d) $\mathbf{K}$ is closed under increasing union of $\omega$-chains.

2) For $\tau, \theta, \mathbf{K}$ as above let $\psi_{\mathbf{K}} \in \mathbb{L}_{\left(2^{\theta}\right)^{+}, \theta^{+}}^{0}(\tau)$ be a sentence such that for $\tau$-models $M$ we have: $M \models \psi_{\mathbf{K}}$ iff for any $A \subseteq M$ of cardinality $\theta$ there is $h: A \rightarrow \omega$ such that for every large enough $n, M\left\lceil h^{-1}\{n\} \in \mathbf{K}\right.$.

Example 4.3. Let $\mathbf{K}_{\theta}^{\mathrm{wo}}=\left\{\left(A,<_{*}\right):<_{*}\right.$ is a linear order of $A$ and for some sequence $\left\langle\mathscr{U}_{n}: n<\omega\right\rangle$ we have $\left(\mathscr{U}_{n},<_{*} \mid \mathscr{U}_{n}\right) \cong(\theta,<)$ and $\mathscr{U}_{n} \subseteq \mathscr{U}_{n+1}$ and $A=\bigcup\left\{\mathscr{U}_{n}\right.$ : $n<\omega\}$.

Claim 4.4. For $\tau, \mathbf{K}$ as in Definition 4.2

1) The sentence $\psi_{\mathbf{K}}$ belongs to $\mathbb{L}_{\left(2^{\theta}\right)^{+}, \theta^{+}}(\tau)$ indeed.

2) If $\theta+|\mathbf{K} / \cong|=\mu$, then moreover $\psi_{\mathbf{K}} \in \mathbb{L}_{\mu^{+}, \theta^{+}}^{0}$.

3) Moreover $\psi_{\mathbf{K}} \in \mathbb{L}_{\leq \theta}^{1}$.

Proof. 1), 2) Obvious.

3) Easy.

Claim 4.5. 1) If $\lambda \geq 2^{\theta}$, then $\Theta_{\theta, R}$ has a model of cardinality $\lambda$ iff $\operatorname{cov}\left(\lambda, \theta^{+}, \theta^{+}, \aleph_{0}\right)$ $=\lambda$; see Remark 4.7 below (or [Sh:g, II]).

2) If $\lambda=\lambda^{\aleph_{0}} \geq 2^{\aleph_{0}}$, then $\Theta_{\theta, R}$ has a model of cardinality $\lambda$ iff $\lambda=\lambda^{\theta}$.

Proof. 1) Read the definitions.

2) Check.

Complementary is

Claim 4.6. Assume $\left(\kappa=\beth_{\kappa}\right.$ and $) \lambda=\operatorname{cov}\left(\lambda, \kappa, \kappa, \aleph_{1}\right)$ and $\tau$ is a vocabulary of cardinality $<\lambda$. For every $\tau$-model $M$ of cardinality $>\lambda$ there is $N \subseteq M$ of cardinality $\lambda$ such that $N \equiv_{\mathbb{L}_{k}^{1}} M$.

2) Assume $\lambda=\operatorname{cov}\left(\lambda, \beth_{\gamma}, \beth_{\gamma}, \aleph_{1}\right)$ for every $\gamma<\theta^{+}$. If $M$ is a $\tau$-model, $|\tau| \leq \beth_{\theta^{+}}$ and $\|M\|>\lambda$, then there is $N \subseteq M$ of cardinality $\lambda$ such that $N \equiv_{\mathbb{L}_{\leq \theta}^{1}} M$.

Proof. Similar to Conclusion 2.15(4)(c), but in $\omega$ stages deal simultaneously with $\lambda$ submodels in each stage.

Remark 4.7. Recall that $\operatorname{cov}(\lambda, \mu, \theta, \sigma)=\operatorname{Min}\left\{|\mathscr{P}|: \mathscr{P} \subseteq[\lambda]^{<\mu}\right.$ and for every $u \in[\lambda]^{<\theta}$ there are $\alpha<\sigma$ and $u_{i} \in \mathscr{P}$ for $i<\alpha$ such that $\left.u \subseteq \bigcup\left\{u_{i}: i<\alpha\right\}\right\}$ for $\lambda \geq \mu \geq \theta \geq \sigma$.

Concerning the upward LST theorem, the logic $\mathbb{L}_{\kappa}^{1}$ fails it badly.

Claim 4.8. There is a sentence $\psi$ from $\mathbb{L}_{\leq \aleph_{1}}^{1}$ such that: $\psi$ has a model of cardinality $\lambda$ iff $\lambda \nrightarrow\left(\aleph_{1}\right)_{2}^{<\omega}$.

Proof. Easy, recalling Claim 4.4(3).

Discussion 4.9. We can phrase other relatives. Also it points to the restrictions when we are looking for such logics with upward LST. 
Theorem 4.10. 1) For every $\theta$ there are models $\left\langle M_{n}, N_{n}: n<\omega\right\rangle$ with a fixed countable vocabulary such that $M_{n}, N_{n}$ are $\mathbb{L}_{\leq \theta}^{1}$-equivalent for $n<\omega$ but $\prod_{n<\omega} M_{n}, \prod_{n<\omega} N_{n}$ are not $\mathbb{L}_{\leq 2^{\aleph_{0}}}^{1}$-equivalent.

2) Moreover in $\partial_{\mathrm{qf}(\tau), 2^{\aleph_{0}}, \omega+1}\left[\prod_{n<\omega} M_{n}, \prod_{n<\omega} N_{n}\right]$ the AIS player has a winning strategy.

Proof. Let $\tau=\{c,<\} \cup\left\{F_{n}: n<\omega\right\}$, where $F_{n}$ is a unary function, $c$ is an individual constant and $<$ is a binary relation.

For every $\alpha$ let $M_{\alpha}^{n}$ be the $\tau$-model with universe $1+\alpha, c^{M_{\alpha}^{n}}=0,<^{M_{\alpha}^{n}}=\{(i, j)$ : $i=0=j$ or $i<j<1+\alpha\}$ and $F_{k}^{M_{\alpha}^{n}}$ is the identity when $k=n$ and is constantly 0 otherwise.

Fixing $\theta \geq 2^{\aleph_{0}}$, by Claim 3.1 for some $\alpha=\alpha(\theta)$ large enough for each $n$ there is a model $N_{n}$ which is $\mathbb{L}_{<\theta}^{1}$-equivalent to $M_{\alpha}^{n}$, but $<{ }^{N_{n}} \uparrow\left(\left|N_{n}\right| \backslash\left\{c^{N_{n}}\right\}\right)$ is not well ordered; let $M_{n}=M_{\alpha}^{n}$ and $\left\langle d_{n, k}: k<\omega\right\rangle$ be a $<^{N_{n}}$-decreasing sequence of members of $N_{n}$ which are $\neq c^{N_{n}}$.

Let $M=\prod_{n<\omega} M_{n}$ and $N=\prod_{n<\omega} N_{n}$. For $\eta \in{ }^{\omega} \omega$ let $d_{\eta}=\left\langle d_{n, \eta(n)}: n<\omega\right\rangle \in$ $\prod_{n<\omega} N_{n}=N$.

Now

$\circledast$ if $\Lambda \subseteq{ }^{\omega} \omega$ is non-meagre or just not bounded in $\left({ }^{\omega} \omega,<_{J}\right.$ bd $)$, then there is no partial isomorphism $f$ from $N$ into $M$ with domain $\left\{\stackrel{\omega}{d}_{\eta}: \eta \in \Lambda\right\}$; i.e. $f$ should preserve all quantifier-free formulas.

This clearly suffices as if $\Lambda=\bigcup\left\{\Lambda_{n}: n<\omega\right\}$, then at least for one $n$, the set $\Lambda$ is non-meagre (or just unbounded in $\left({ }^{\omega} \omega,<_{J_{\omega}}\right.$ d $)$ ).

Why is $\circledast$ true? Toward a contradiction assume that $f$ is such a partial isomorphism. By the assumption for some $n(*)<\omega$ the set $\{\eta(n(*)): \eta \in \Lambda\}$ is infinite, so choose $\bar{\eta}=\left\langle\eta_{k}: k<\omega\right\rangle$ such that

$(*)_{0}\left\langle\eta_{k}(n(*)): k<\omega\right\rangle$ is strictly decreasing in $N_{n(*)}$.

Now:

$(*)_{1}$ if $m(1)<m(2)<\omega$, then for every $n<\omega$ we have $N_{n} \models " F_{n(*)}\left(d_{n, \eta_{m(2)}(n(*))}\right)$ $<F_{n(*)}\left(d_{n, \eta_{m(1)}(n(*))}\right)$.

[Why? If $n=n(*)$, then $F_{n(*)}^{N_{n}}$ is the identity and this means $N_{n} \models$ " $d_{n, m(2)}<$ $d_{n, m(1)}$ " which holds by the choice of $\left\langle d_{n, k}: k<\omega\right\rangle$ recalling $(*)_{0}$. If $n<\omega \wedge n \neq$ $n(*)$, then $F_{n(*)}^{N_{n}}$ is constantly 0 , so this means that $N_{n} \models$ " $0<0$ ", which holds by the choice of $N_{n}$ (and $<M_{\alpha}^{n}$ ), so we are done.]

$(*)_{2} \quad N \models " F_{n(*)}\left(d_{\eta_{m(2)}}\right)<F_{n(*)}\left(d_{\eta_{m(1)}}\right)$ " for $m(1)<m(2)<\omega$.

[Why? By $(*)_{1}$ and the definition of products.]

Also:

$(*)_{3}$ If $m<\omega$, then $N_{n(*)} \models$ "c $\neq F_{n(*)}\left(d_{n(*), \eta_{m}(n(*))}\right)$ "; hence $N \models$ "c $\neq$ $F_{n(*)}\left(d_{\eta_{n}}\right) "$.

$(*)_{4}$ Let $\nu_{k}=f\left(d_{\eta_{k}}\right)$. Then $\nu_{k}=\left\langle a_{k, n}: n<\omega\right\rangle \in \prod_{n<\omega} M_{n}$. 
But $f$ is a partial isomorphism, so by $(*)_{2}+(*)_{3}+(*)_{4}$ we have

$$
(*)_{5} \quad M \models " F_{n(*)}\left(\nu_{m(2)}\right)<F_{n(*)}\left(\nu_{m(1)}\right) \neq c \text { " for } m(1)<m(2)<\omega .
$$

Hence for every $n, M_{n} \models " F_{n(*)}\left(\nu_{m(2)}(n)\right)<F_{n(*)}\left(\nu_{m(1)}(n)\right)$ " for $m(1)<m(2)<$ $\omega$. Also by the choice of $F_{n(*)}^{M_{n}}$ we have $n \neq n(*) \Rightarrow M_{n} \models$ "c $=F_{n(*)}\left(\nu_{m(1)}(n)\right)$ " but $M \models " c \neq F_{n(*)}\left(\nu_{m(1)}\right)$ "; hence $M_{n(*)} \models$ "c $\neq F_{n(*)}\left(\nu_{m(1)}(n(*))\right)$ ", i.e. $\nu_{m(1)}(n(*))$ $\neq 0$. Together $\left\langle\nu_{m}(n(*)): m<\omega\right\rangle$ is $<^{M_{n}}$-decreasing in $M_{n(*)} \backslash\{0\}$, a contradiction.

\section{REFERENCES}

[Be85] Jon Barwise and Solomon Feferman (editors), Model-theoretic logics, Perspectives in Mathematical Logic, Springer-Verlag, Heidelberg-New York, 1985. MR819531 (87g:03033)

[Dic85] M. A. Dickman, Larger infinitary languages, Model Theoretic Logics (J. Barwise and S. Feferman, eds.), Perspectives in Mathematical Logic, Springer-Verlag, New York, Berlin, Heidelberg, Tokyo, 1985, pp. 317-364. MR819540

[EM02] Paul C. Eklof and Alan Mekler, Almost free modules: Set theoretic methods, NorthHolland Mathematical Library, vol. 65, North-Holland Publishing Co., Amsterdam, 2002, Revised Edition. MR.1914985 (2003e:20002)

[FV59] S. Feferman and R.L. Vaught, The first order properties of products of algebraic systems, Fund. Math. 47 (1959), 57-103. MR.0108455 (21:7171)

[Kop85] Sabine Koppelberg, Homogeneous Boolean algebras may have nonsimple automorphism groups, Topology and its Applications 21 (1985), 103-120. MR813282 (87a:06030)

[Mak85] Johann A. Makowsky, Compactnes, embeddings and definability, Model-Theoretic Logics (J. Barwise and S. Feferman, eds.), Springer-Verlag, 1985, pp. 645-716. MR819549

[Mos52] Andrzej Mostowski, On direct products of theories, J. Symbolic Logic 17 (1952), 1-31. MR0047574 (13:897a)

[Rv89] Matatyahu Rubin and Petr Štěpánek, Homogeneous Boolean algebras, Handbook of Boolean algebras, vol. 2, North-Holland, Amsterdam, 1989, pp. 679-715. MR.991606

[Sh:b] Saharon Shelah, Proper forcing, Lecture Notes in Mathematics, vol. 940, SpringerVerlag, Berlin-New York, xxix+496 pp., 1982. MR675955 (84h:03002)

[Sh:g] _ Cardinal Arithmetic, Oxford Logic Guides, vol. 29, Oxford University Press, 1994. MR1318912 (96e:03001)

[Sh:12] _ The number of non-isomorphic models of an unstable first-order theory, Israel Journal of Mathematics 9 (1971), 473-487. MR0278926 (43:4652)

[Sh:18] _ On models with power-like orderings, Journal of Symbolic Logic 37 (1972), 247-267. MR0446955 (56:5272)

[Sh:72] ㄴ Models with second-order properties. I. Boolean algebras with no definable automorphisms, Annals of Mathematical Logic 14 (1978), 57-72. MR.501097 (80b:03047a)

[Sh:199] , Remarks in abstract model theory, Annals of Pure and Applied Logic 29 (1985), 255-288. MR808815 (87g:03040)

[Sh:384] C Compact logics in ZFC: Complete embeddings of atomless Boolean rings, Non structure theory, Chapter X.

[FuSh:766] Laszlo Fuchs and Saharon Shelah, On a non-vanishing Ext, Rend. Sem. Mat. Univ. Padova 109 (2003), 235-239, math.LO/0405015. MR.1997989

[Sh:800] Saharon Shelah, On complicated models, Preprint.

[GbSh:880] Ruediger Goebel and Saharon Shelah, Absolutely Indecomposable Modules, Proceedings of the American Mathematical Society 135 (2007), 1641-1649, arXiv 0711.3011. MR2286071 (2007k:13047) 
[GbHeSh:948] Ruediger Goebel, Daniel Herden, and Saharon Shelah, Absolute E-rings, Advances in Mathematics, accepted.

[Sh:F1046] Saharon Shelah, Nice infinitary logics II.

Einstein Institute of Mathematics, Edmond J. Safra Campus, Givat Ram, The Hebrew University of Jerusalem, Jerusalem, 91904, Israel

Current address: Department of Mathematics, Hill Center - Busch Campus, Rutgers, The State University of New Jersey, 110 Frelinghuysen Road, Piscataway, New Jersey 08854-8019

E-mail address: shelah@math.huji.ac.il

URL: http://shelah.logic.at 